\title{
Diagnostics for magnetically confined high-temperature plasmas
}

\author{
K. W. Gentle \\ Fusion Research Center, University of Texas, Austin, Texas 78712
}

\begin{abstract}
During the last 20 years, magnetically confined laboratory plasmas of steadily increasing temperatures and densities have been obtained, most notably in tokamak configurations, and now approach the conditions necessary to sustain a fusion reaction. Even more important to the goal of understanding the physics of such systems, remarkable advances in plasma diagnostics, the techniques for determining the properties of such plasmas, have accompanied these developments. More parameters can be determined with greater accuracy and finer spatial and temporal resolution. The magnetic configuration, the primary local thermodynamic quantities (density, temperature, and drift velocity), and other necessary quantities can now be measured with sufficient accuracy to determine particle and energy fluxes within the plasma and to characterize the basic transport processes. These plasmas are far from thermodynamic equilibrium. This deviation manifests itself in a variety of instabilities on several spatial and temporal scales, many of which are aptly described as turbulence. Many aspects of the turbulence can also be characterized. This article reviews the current state of diagnostics from an epistemological perspective: the capabilities and limitations for measuring each important physical quantity are presented.
\end{abstract}

\section{CONTENTS}

I. Introduction

II. Framework for Description

A. Ideal magnetohydrodynamic equilibrium

B. Resistive magnetohydrodynamics and instabilities

C. Transport

D. Fluctuations and turbulence

E. Other effects

F. Time and space scales

III. Equilibrium Magnetic Configuration

A. Global properties

B. Circular cross sections

C. Noncircular cross sections

IV. Determining the Local Fluid Variables
A. Density
B. Electron temperature
C. Ion temperature
D. Velocity
E. Current density

V. Transport

A. Energy

B. Particles

C. Momentum

D. Time-dependent transport

VI. Fluctuations
A. Density
B. Other
C. Transport

VII. Kinetic Effects

VIII. Asymmetries and Edge Effects

IX. Conclusion

Acknowledgments

References

\section{INTRODUCTION}

The recent experiments with deuterium-tritium plasmas in JET (at Culham, Great Britain) (JET Team, 1992) and TFTR (at Princeton Plasma Physics Laboratory, Princeton, NJ) (Strachan et al., 1994) have publicized the fact that laboratory plasmas have nearly reached the conditions necessary for sustained thermonuclear fusion. The plasmas have certainly attained the regime of relevant physics, as gauged by dimensionless parameters, for example. The progress toward fusion parameters of the past two decades has been paralleled by improvements in our understanding of hot plasmas. Essential to this development, and arguably the principal contributor, have been the impressive advances in techniques for measuring plasma properties, collectively referred to as "plasma diagnostics."

The description of laboratory plasmas has undergone a qualitative change over the last decade. Formerly, a discharge was characterized only by gross global or average parameters, e.g., total energy content, energy confinement time, and average density. There was little local information and no possibility of ascertaining the physical processes which determined the observed results. Nowadays, the equilibrium internal structure of the plasma may be determined and the major parameters, like density, temperature, and fluid velocity, found as a function of position. As a result, inferences of particle, energy, and momentum flux can be drawn. The fluxes are generally found to be "anomalous" in the sense that they greatly exceed those which would be implied by the basic collisional processes in the plasma, the fluxes calculated from neoclassical transport theory. The fluxes are presumed to be driven by some form of plasma turbulence, fluctuations on a scale much smaller than the size of the plasma. Techniques for measuring these fluctuations, especially in density, have made notable progress.

All plasma measurements are intrinsically difficult, for the high temperatures preclude the use of any solid material measuring instrument. The techniques must be indirect, being made from outside the plasma. They may be passive, using the natural emission of radiation or particles, or they may be active, observing the effect of the plasma on a source of applied radiation or particles; but the interaction must be weak in the sense of not perturbing the system. There is an extensive literature for each technique, and many have involved the development of extensive physics in their own rights. For the practitioner, diagnostics may be conveniently organized by these measuring processes, e.g., spectroscopy, interferometry, etc. However, a different approach will be taken here.

This review will take the perspective of the physicist studying the plasma. First, a general framework for analysis 
will be presented, beginning with the fundamental magnetohydrodynamic (MHD) equilibrium for an axisymmetric system, continuing with elementary MHD stability and neoclassical transport, and concluding with microinstabilities. Then, for each of these aspects, the physical quantities that must be measured will be enumerated and the state of the measuring art evaluated. In addition, various limitations, weaknesses, and approximations in the basic framework will be indicated, along with the related diagnostic implications. Representative references are cited, but the emphasis is on the results provided by a technique rather than on the application and development of the technique itself. A full survey of the literature on diagnostic development would be awkwardly long, and it would duplicate many excellent reviews of specific techniques. Not even a comprehensive survey of techniques is possible within this compass. In emphasizing the measurement of the principal physical parameters, only the primary techniques for determining each will be reviewed. Many valuable techniques which have been developed and applied as alternatives to the primary techniques, often with specific advantages in certain circumstances, are omitted here. Likewise, many types of experiments that have contributed important information on plasma behavior are omitted here because they are not routinely employed as diagnostics.

This review focuses on tokamaks. Since all diagnostic techniques are in some sense applicable to any hot plasma, this focus is more a matter of convenience in exposition than of necessity. However, specifics of emphasis, interpretation, and accuracy are often dependent on magnetic configuration. As the dominant configuration, the tokamak is the natural choice. However, the extreme contrast in temporal and spatial scales between magnetically confined and inertial fusion plasmas provides little overlap in diagnostic practice. Diagnostics for many other laboratory plasmas, as used for materials processing or astrophysical simulation, etc., are also omitted here. The objective here is to demonstrate how a great many diagnostic developments have combined to illuminate the detailed physics of hot, magnetically confined plasmas. This is not, however, a review of tokamak physics. Only a few aspects of contemporary research are included, and those because they have important implications for diagnostics. Likewise, the continuing efforts at improving diagnostics and the exciting new physics that is appearing are beyond the scope of this review.

In addition to the specific citations given for each topic, more general, complete expositions are available in several books. Krall and Trivelpiece (1973) remains a good basic reference. Hazaltine and Meiss (1992) provides a more advanced treatment of magnetic confinement; and Wesson (1987) is an excellent compilation of various aspects of tokamak physics and is also valuable for its annotated bibliography. Hutchinson (1987) is valuable as a general diagnostic reference. As a recent review of the state of the art in diagnostic development, the conference proceedings edited by Stott, Akulina, Gorini, and Sindoni (1991) is useful.

\section{FRAMEWORK FOR DESCRIPTION}

\section{A. Ideal magnetohydrodynamic equilibrium}

A hot plasma may be usefully and accurately described as a perfectly conducting fluid. The justification of fluid equa-

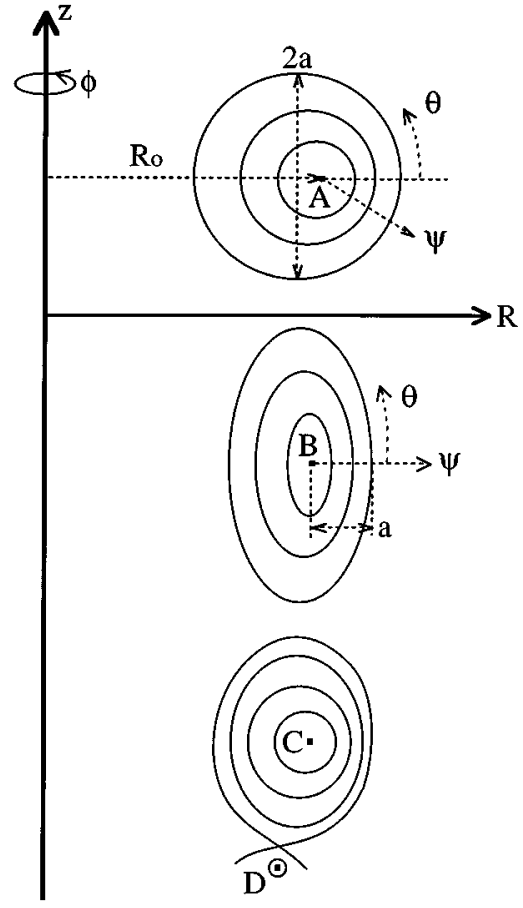

FIG. 1. Coordinates for magnetic configuration. $A, B$, and $C$ are the magnetic axes of typical configurations - circular, elongated, and diverted. The cross sections of three closed flux surfaces around the axis are shown in each case.

tions is not trivial, for collisions are insufficiently frequent to support the usual analyses of molecular fluids. The approximations of isotropic pressure $p$ and zero resistivity limit the validity. Nonetheless, ideal MHD is a useful approximation and an invaluable starting point (Kruskal and Kulsrud, 1958). Insofar as one is interested in a hot plasma that continues for some significant period of time, stable solutions of the ideal MHD equations are a self-consistent necessity. The equilibrium equations are simply force balance and Maxwell's equations for the magnetic field $\mathbf{B}$ and current density j:

$$
\mathbf{j} \times \mathbf{B}=\nabla p ; \quad \nabla \cdot \mathbf{B}=0 ; \quad \nabla \times \mathbf{B}=\mu_{0} \mathbf{j} .
$$

It follows immediately that

$$
\mathbf{B} \cdot \nabla p=0,
$$

that the pressure gradient must be everywhere perpendicular to the magnetic field. The necessary pressure gradient for a hot plasma can therefore only be sustained if the magneticfield lines lie on a set of nested surfaces. Spheres being inadmissible for $\mathbf{B}$, the topology must be that of nested tori. These configurations are generally described with variants of cylindrical coordinates as illustrated in Fig. 1. The usual laboratory coordinates are the conventional $R, z$, and $\phi$, but the magnetic configurations are best described with respect to the magnetic axis. Three representative configurations are shown in Fig. 1, with the magnetic axes labeled $A, B$, and $C$. A coordinate $\psi$, which plays the role of minor radius for the tori, labels the magnetic-flux surfaces, and a poloidal angle variable $\theta$ specifies position on the flux surface in an $R, z$ plane. Although the simple polar $\theta$ is used for some purposes, the theory is most conveniently developed in a 
rather complicated, nonorthogonal system in which the fluxsurface label $\psi$ is the toroidal flux enclosed, and $\theta$ is also defined by flux. The importance and utility of this description arise from the fact that the pressure is constant on a flux surface and varies only with $\psi$. Since thermal conductivity along magnetic-field lines is also excellent, the temperature $T$ and thus the density $n$ are also functions only of $\psi$. Many aspects of plasma behavior can be treated in one dimension as a function only of $\psi$.

For the important case of axisymmetry, e.g., a tokamak, in which all dependencies on the toroidal angle $\phi$ vanish, the task of solving Eqs. (2.1) for the magnetic configuration can be reduced to that of solving the single scalar GradShafranov equation (Shafranov, 1966; Grad, 1967) for the flux surfaces $\psi(R, z)$ :

$$
\begin{aligned}
& \Delta^{*} \psi=-I \frac{d I}{d \psi}-\mu_{0} R^{2} \frac{d p}{d \psi} ; \\
& \Delta^{*} \psi \equiv R^{2} \nabla \frac{\nabla \psi}{R^{2}} .
\end{aligned}
$$

The solution for $\psi(R, z)$ is determined by the two independent functions $p(\psi)$ and $I(\psi)$, which are profiles of pressure and toroidal current, and boundary conditions outside the plasma, where the right-hand side of Eq. (2.3) becomes zero. The condition of axisymmetry applies to several magnetic configurations important for fusion plasmas, both tokamaks and reversed-field pinches, but not to all (e.g., stellarators). Related, albeit more complicated, equations are needed to obtain $\psi(R, z, \phi)$ for such cases. The solutions of Eq. (2.3) are fundamental to all tokamak diagnostics because so many plasma parameters are either exactly or approximately fluxsurface variables-functions only of $\psi$. The complexity arises because this natural coordinate system for describing the plasma cannot be chosen a priori, but is only determined implicitly by Eq. (2.3) in terms of pressure and current profiles, which are assumed given in Eq. (2.3) but which must be either obtained experimentally or determined by a selfconsistent analysis of the plasma. In general, the GradShafranov equation must be solved numerically, for which some references will be given in Sec. III; but an approximate solution can be obtained in one limiting case of considerable practical utility. If the plasma is small in the sense that its minor radius ( $a$ in Fig. 1 ) is much less than the major radius $R_{0}$ of its magnetic axis, a complete approximate solution may be obtained for which the flux surfaces have nested circular cross sections, but with centers shifted from the magnetic axis. The magnetic axis $A$ in Fig. 1 illustrates this solution. More complicated solutions occur for larger plasmas or for more complicated boundary conditions. As illustrated for magnetic axis $C$, the presence of toroidal current $D$ can even change the magnetic topology. The plasma here terminates at the separatrix, the magnetic surface shown with the $X$ point singularity. Magnetic-field lines outside the separatrix intersect solid surfaces, and plasma is lost rapidly. These are called divertor configurations. Regardless of configuration, $R_{0}$ is the major radius of the magnetic axis, and $a$ is a measure of plasma size, as indicated in Fig. 1.

Individual diagnostic instruments use a variety of coordinates to represent their observations. Along sight lines in the midplane, minor radius $f(r)$ or major radius $f(R)$ may be used. For vertical views, $f(z)$ is convenient. However, for a comparison of results from different views and for further analysis, a flux-surface representation $f(\psi)$ is needed. The usual choice is a variable $\rho(\psi), 0 \leqslant \rho \leqslant 1$ for the closed flux surfaces such that $\rho=r / a$ for the simple circular case and is extrapolated in a reasonable way to more complex cases. As a dimensionless variable, it is also useful for comparisons between different devices. It will be used here extensively to represent radial $(\psi)$ variations.

The experimentally relevant solutions of the GradShafranov equation must be stable in the context of ideal MHD. Choices of $p(\psi), I(\psi)$, or boundary condition which led to unstable solutions would have scarcely been seen; the ideal instabilities would grow and destroy the configuration too quickly. For tokamaks, the constraint is primarily one on $I(\psi)$, the plasma current, and requires that the quantity $q>1$ at the boundary. The $q$ describes how the field lines move on the magnetic surface and is proportional to the ratio between the poloidal $(\theta)$ and toroidal $(\phi)$ components of the field. If $q$ is a rational number, $q=m / n$, the field lines close after making $m$ circuits around the torus, having made $n$ circuits poloidally. These rational surfaces have special significance, especially for small $m, n$, the low-order surfaces. The requirement that $q>1$ (in practice, $q \geqslant 2$ ) implies that the toroidal component of magnetic field, produced largely by external field coils in a tokamak, must substantially exceed the poloidal component, which is produced by the toroidal current in the plasma.

The literature on MHD, both the ideal of this section and resistive of Sec. II.B, is extensive even if one restricts consideration to review articles. It is most accessible through one of a number of excellent monographs: Bateman (1978), Freidberg (1987), and Manheimer and Lashmore-Davies (1989).

\section{B. Resistive magnetohydrodynamics and instabilities}

The more interesting stability considerations appear when the constraint of perfect conductivity is relaxed and resistive MHD equations are employed. Although one might expect the introduction of dissipation to stabilize a system, perfect conductivity is actually a constraint, preserving magnetic flux. Finite resistivity releases the constraint and permits additional classes of perturbations, which can be unstable. In this sense, the consideration of resistive MHD is essential, even for hot plasmas of very high conductivity. The theory of plasma stability based on the resistive MHD equations is extensive, complex, and far beyond the scope of this paper. However, several results are important for diagnostics. First, the solutions for static equilibria are not changed; the implications are largely for stability.

Second, the instabilities arise on rational magnetic surfaces, often the low-order ones. (Rational surfaces are vulnerable because one can have a different current on each closed field line without violating $\nabla \cdot \mathbf{j}=0$.) The instabilities involve a change in the magnetic topology and modification of the simple set of nested surfaces as in Fig. 1. Additional magnetic axes may develop with separatrices inside the plasma, and localized regions of chaotic field lines, lacking 
magnetic surfaces, may even develop. The instabilities are secular, simply growing in time without oscillation; but they have a spatial structure determined by the $m, n$ of the rational surface, $m$ periods poloidally and $n$ periods toroidally. Since laboratory plasmas generally rotate, these instabilities and structures are typically observed as oscillations by a fixed instrument.

Finally, laboratory plasmas have generally been found to be stable or at the stability boundary for resistive MHD modes. Some modes and structures are found, especially associated with $q=1$ and 2 surfaces inside the plasma; but for the plasmas of greatest interest, the effects are either absent or localized. The bulk of the plasma may be described by the ideal static equilibrium magnetic configuration.

A modest qualification is the phenomenon of sawtooth oscillations. These are relaxation oscillations in which the central temperature slowly rises and abruptly falls (a sawtooth). Outside the core, presumed to be bounded by the $q=1$ surface, the temperature rises when the central temperature falls. Aside from their intrinsic physics interest, sawteeth have several diagnostic implications. As oscillations with periods of one to several hundred milliseconds, depending upon machine and conditions, they represent a possible complication to simple, static analyses. On the other hand, they are helpful in indicating $q \sim 1$, and the propagation of the temperature pulse outward through the plasma is useful for transport analysis (cf. Sec. V.D).

\section{Transport}

The Grad-Shafranov equation assumes that the current and pressure profiles are given, but of course they are only indirectly controlled by the experimentalist. In most tokamaks, the toroidal current is driven by using the plasma as a oneturn secondary of a transformer, which does allow control of the total current, but not the distribution. The pressure is subject to even less control. In the simplest tokamaks, the only energy input is through the resistive dissipation of the plasma current, Ohmic heating, and is thus not independently adjustable. Most modern tokamaks have additional sources of heating - neutral beams or rf heating - which provide independent control of the total energy input, but limited control over its deposition. The pressure is largely determined by the internal plasma transport processes.

Within the context of ideal MHD, an equilibrium state is perfectly confined; there is no energy loss or transport. This is not altered by the introduction of resistivity. If the configuration is stable, there is no transport. (Only if the system were linearly unstable and were to evolve nonlinearly to some sort of fluctuating quasi-steady state would the possibility of a transport process arise.) Transport does arise from collisions, which must be introduced in a kinetic description of the plasma. The problem is far more difficult than Chapman-Enskog theory because collisions are infrequent and the deviations from a local Maxwellian more extensive. The pertinent analysis, neoclassical transport theory, is again extensive, complex, and far beyond the scope of this paper (for a review, see Hinton and Hazeltine, 1976). Fortunately, only the form of the result is essential for plasma diagnostics.

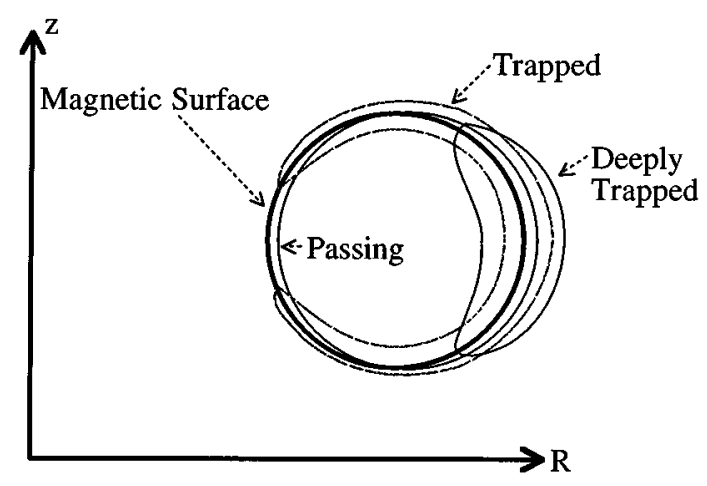

FIG. 2. Representative guiding-center trajectories in the $R, z$ plane in a tokamak. The trajectories correspond to very energetic ions; typical thermal trajectories lie much closer to the magnetic surface.

Each species-electrons, hydrogen ions, and each additional ion species-must be considered separately. The particle trajectories in the absence of collisions are complicated. The particles circle the field lines at the gyrofrequency, $\Omega=e B / M$, with an average radius $\rho_{e, i}=v_{\mathrm{th}} / \Omega$ $=\sqrt{k T / M} / \Omega$ for each species. The subscripts $e, i$ will be used to distinguish electrons and ions, for example $v_{e}$ th and $v_{i \text { th }}$ for the thermal velocities, although $m$ and $M$ are also used conventionally for electron and ion masses. The trajectory of the center of this circle (the guiding center) generally follows the field line, and the guiding-center orbit lies close to the magnetic surface. The magnetic field in the tokamak is dominated by the toroidal field with a $1 / R$ dependence on major radius. The conservation of magnetic moment, $\mu=M v_{\perp}^{2} / B$, implies that those particles with a small ratio of parallel to perpendicular energy on the outside where $B$ is low will not be able to follow field lines to the inside where $B$ is high. Their perpendicular energy would have to increase at the expense of parallel energy, which is not available. This is a form of magnetic mirror trapping that leads to banana trajectories as illustrated in Fig. 2. Figure 2 shows typical guiding-center trajectories for both passing (untrapped) and banana (trapped) particles. For visibility, the deviations from the magnetic surface are exaggerated. The ion excursions in modern tokamaks are typically a few percent of the minor radius, and the electron excursions an additional factor of 40 smaller. These are effects that complicate neoclassical theory and lead to significant variations over magnetic surfaces. However, appropriate flux-surface averages can be taken for each species and expressions for the net transport fluxes across magnetic surfaces obtained. Transport analysis can be reduced to a one-dimensional problem in the coordinate perpendicular to magnetic-flux surfaces, $\psi$. This coordinate is easily related to the (minor) radius $r$ for the simple magnetic configuration $A$ of Fig. 1, but it requires some care in more complicated configurations like $B$. An important, general caveat for all magnetically confined systems is that fluid equations and local thermodynamic variables are only definable averaged on a finite scale, here the banana width, which is not dramatically smaller than the gradient scale lengths, e.g., $L_{T}=T /(d T / d r)$ for temperature or $L_{n}=n /(d n / d r)$ for density. Hot plasmas are further from thermodynamic equilibrium than any other physical system. 
For a pure hydrogen plasma with only density, the electron and ion temperatures, and current considered and with rotation ignored (cf. Sec. V.C), a minimal neoclassical transport matrix for the fluxes can be written in the following form:

$$
\left(\begin{array}{c}
\Gamma \\
Q_{e} \\
Q_{i} \\
j_{\|}
\end{array}\right)=-\left(\begin{array}{cccc}
D & n V_{e} & n V_{i} & n V_{N C} \\
q_{e \text { Conv }} & \kappa_{e} & q_{p e i} & q_{e E} \\
q_{i \text { Conv }} & q_{p e i} & \kappa_{i} & q_{i E} \\
j_{B n} & j_{B e} & j_{B i} & \sigma
\end{array}\right)\left(\begin{array}{c}
\partial n / \partial r \\
\partial T_{e} / \partial r \\
\partial T_{i} / \partial r \\
E_{\|}
\end{array}\right)
$$

where $\Gamma$ is the radial particle flux, (charge neutrality requires identical ion and electron fluxes and densities), $Q_{e}$ and $Q_{i}$ are the electron and ion radial thermal fluxes, and $j_{\|}$is the current along the magnetic fields. The electrons and ions must be treated separately because, although collisions are sufficient to maintain the distribution function of each species near a Maxwellian, collisional energy exchange between species is often too weak to maintain $T_{e}=T_{i}$. The thermodynamic forces on the right are the gradients in density and temperature and the toroidal electric field applied by the external transformer coils $\left(E_{\phi} \sim E_{\|}\right)$. The diagonal terms in the matrix are familiar as diffusion and thermal-conductivity coefficients and electrical conductivity. However, there are also significant off-diagonal terms. For example, the Ware pinch $V_{N C}$ (Ware, 1970) is an inward particle convection driven by the toroidal electric field, and the $j_{B}$ terms are "bootstrap" currents driven by gradients. One could argue that this is not the "correct" choice of thermodynamic forces-it certainly is not the set for which Onsager symmetries in neoclassical theory would apply - but it is an experimentally useful one. Since the observed fluxes generally exceed neoclassical predictions considerably through processes which remain to be determined, the "correct" choice is not yet clear. The paradigm of Eq. (2.4) remains useful for reference and discussion. Extensions for additional ion species and momentum are straightforward, although clearly the matrices become cumbersome to write, not to mention calculate.

The experimental estimation of transport coefficients involves coupling a form like Eq. (2.4) to an appropriate conservation equation for a quantity (density, electron energy, etc.) and measuring all the terms. This will be discussed in Sec. V.

\section{Fluctuations and turbulence}

Since on the one hand transport rates are observed to be much greater than neoclassical and on the other hand hot plasmas are calculated to be unstable to a wide variety of small-scale fluctuations, fluctuations and turbulence must be important phenomena. More sophisticated treatments of a plasma, including at least two fluids and generally some kinetic considerations, introduce numerous instabilities that are unique to inhomogeneous plasmas. It is not that the conventional wave modes, electromagnetic or acoustic, become unstable, but rather that additional modes, which exist only in the presence of density or temperature gradients, are unstable. The modes have small scales and short wavelengths, typically of the order of the ion gyroradius. Much of the original work is reviewed in Kadomtsev and Pogutse (1975) and Tang (1978).

Representative of these instabilities is the drift wave, for which the dispersion relation is

$$
\omega=k_{\theta} v_{d e} ; \quad v_{d e}=\rho_{e} v_{e} \text { th } \frac{1}{n} \frac{d n}{d r}
$$

with phase velocity termed the electron drift velocity and with instability extending over $0<k \rho_{i} \leqslant 1$ in the most simple cases. Such modes clearly require a density gradient for their existence and have low frequency. Evaluation of Eq. (2.5) for typical tokamak parameters implies frequencies below 1 MHz. There are a number of related modes with the generic property of existing only in the presence of a gradient.

These instabilities remain a major topic of research. Linear stability analysis in the appropriate approximation and configuration is difficult. For unstable modes, the nonlinear evolution must be followed to determine the consequences. As with the other theoretical developments, the details are beyond the scope of this article. Liewer (1985) provides a contemporaneous review of both theory and observation. Fortunately, for diagnostic purposes, it is only necessary to know the temporal and spatial scales of the fluctuating quantities and the physical consequences to be expected.

The presence of fluctuations in the fluid variables will generally cause fluxes that can be expressed as follows [see, for example, Wootton et al. (1990) for a discussion, review, and detailed references]:

$$
\Gamma_{s}=\frac{\left\langle\tilde{E}_{\theta} \tilde{n}_{s}\right\rangle}{B_{\phi}}-\frac{\left\langle\tilde{j}_{\| s} \tilde{b}_{r}\right\rangle}{e B_{\phi}},
$$

where $\Gamma_{s}$ is the radial particle flux of species $s$ (electron, ion, or impurity), $\langle\cdots\rangle$ denotes time average, the tilde indicates the time-dependent component of a quantity, and $\tilde{b}_{r}$ is the fluctuating component of the radial magnetic field. The first term is familiar as the electrostatic flux that arises from the $E_{\theta} X B_{\phi}$ radial drift velocity. The second term arises from flows along the magnetic field, which have a radial component if $\tilde{b}_{r}$ is present. The corresponding energy flux is

$$
Q_{s}=\frac{\frac{3}{2} n_{s}\left\langle\tilde{E}_{\theta} k \tilde{T}_{s}\right\rangle}{B_{\phi}}+\frac{\frac{3}{2} k T_{s}\left\langle\tilde{E}_{\theta} \tilde{n}_{s}\right\rangle}{B_{\phi}}+g_{s}\left(\frac{\tilde{b}_{r}}{B_{\phi}}\right) \nabla T_{s},
$$

where the function $g_{s}$ depends on species and the structure of the magnetic turbulence. For weak turbulence at low collisionality,

$$
g_{e} \approx \pi R v_{e \mathrm{th}}\left(\frac{\tilde{b}_{r}}{B_{\phi}}\right)^{2}
$$

as one example, but the function depends on collisionality and the statistical properties of turbulence, which can only be inferred from a correct nonlinear theory or from much more subtle observations. In general, the contribution from magnetic fluctuations would be significant only for electron energy transport, where the large value of $v_{e \text { th }}$ enhances the effect. The significance of Eqs. (2.6) and (2.7) for diagnostics is that they exhibit the quantities that must be measured to infer turbulent fluxes experimentally. The applications will be discussed in Sec. VI. 


\section{E. Other effects}

There is much other physics that is important for tokamaks, especially the atomic physics of impurities and the surface physics of materials at the plasma boundary. Historically, the ascendancy of the tokamak configuration was largely due to the creation of a pure hydrogen plasma and the reduction in impurity content. The continuing gains in performance have depended upon improvements in boundary conditions and wall conditioning to maintain pure plasmas. Spectroscopic measurements of impurities are necessary for effective tokamak operation. Further discussion can be found in Hutchinson (1987) and Wesson (1987). Although these diagnostics have great technical importance, they are not fundamental to the understanding of tokamak physics and will not be emphasized here. However, two further subtleties in the analysis of the plasma, kinetic effects and asymmetries, do have fundamental diagnostic implications.

The plasma is generally described by fluid equations, either the single fluid of MHD or coupled electron, ion, and, possibly, impurity fluids, but without consideration of a distribution function or higher moments. In addition to the previously mentioned calculations of neoclassical theory and plasma turbulence, kinetic effects are generally important for plasma heating, processes which impart energy to the plasma. Even for Ohmic heating, collisions are insufficient to maintain a Maxwellian electron distribution. A small number of electrons (runaways) accelerate freely and escape the magnetic confinement with $\mathrm{MeV}$ energies. At low densities, even stronger deviations occur. Electromagnetic heating at the electron cyclotron frequency $(\mathrm{ECH})$ requires kinetic analysis to calculate the energy absorption and can cause significant non-Maxwellian distributions.

Kinetic effects for ions are even more general. Heating with neutral beams directly introduces a large nonMaxwellian component in the ion distribution, and rf heating at the ion cyclotron frequency (ICRH) can produce nonMaxwellian tails. Near the plasma edge, collisions can be insufficient to maintain an average Maxwellian between the cold ions from ionization and the hot ions diffusing from the core. These effects require a kinetic analysis, but, more important to the present purpose, imply that measurement of the full distribution function is an important objective.

Thus far, two major symmetries have been assumed. As an axisymmetric concept, the tokamak has been taken as perfectly symmetric in $\phi$, and the constancy of pressure on flux surfaces has been implicitly extended to poloidal symmetry and $\theta$ independence, leaving only radial or $\psi$ variations. Practically, the symmetry can be only approximate, and some of the deviations are significant. Toroidally, the major internal effect arises from the discrete field coils, which produce ripples in the toroidal field, leading to trapping and ion kinetic effects. At the edge, boundary conditions rarely have toroidal symmetry. Localized limiters, gas feed, antenna structures, etc., all introduce asymmetries. These may have significant effects on edge behavior and thus on global properties; but from a diagnostic viewpoint, these toroidal asymmetries are localized to the edge and do not persist into the interior.
The poloidal direction is much more complicated. Only for the simplest case of roughly circular flux surfaces (Fig. 1, axis $A$ ) and weak plasma pressure effects is there symmetry in the polar coordinate $\theta$. Otherwise, although the pressure is uniform, other quantities may vary over the flux surface. The generally fast transport along field lines precludes sharp variations over a magnetic surface, but diagnostics must treat poloidal variations. The edge region poses more severe problems, for the boundary conditions are often highly asymmetric poloidally. The poloidal asymmetries generally decay less rapidly toward the plasma interior than do toroidal ones.

\section{F. Time and space scales}

To conclude this outline of tokamak physics, it is useful to summarize the various characteristic times and lengths. These govern the technical requirements for all diagnostics and determine the spatial and temporal resolution required to examine various phenomena. The principal parameters are listed in Table I.

The typical values listed in Table I are only intended to give an indication of the order of magnitudes of the various quantities and do not indicate the range of values encountered in the many extant tokamaks or even the spatial variation within a single device. Most of the quantities listed are well known and have been discussed previously, but the transport times require a few comments.

From the transport matrix of Eq. (2.4), one could take the particle diffusivity $D$ and the plasma size $a$ to define a characteristic time $a^{2} / D$ for density equilibration. Similarly, one could use the thermal diffusivity $\kappa_{e}=n \chi_{e}$ to obtain a thermal equilibration time $a^{2} / \chi_{e}$. However, an experimental measure, the energy confinement time $\tau_{E}$, has been established by long usage and serves quite well. The definition is basically

$$
\tau_{E}=\frac{\text { plasma energy content }}{\text { power input }}=\frac{\int n\left(T_{e}+T_{i}\right) d V}{P_{\text {in }}},
$$

where originally the input power was simply the Ohmic power, $P_{\text {in }}=\mathrm{V}_{\text {loop }} \mathrm{I}_{\text {plasma }}$. The literature includes many variations of Eq. (2.9), depending upon whether nonthermal components of plasma energy are included, what estimate of $P_{\text {in }}$ to use for additional heating methods, and what correction to apply if the parameters are changing in time, among others. Regardless, $\tau_{E}$ is a good estimator of the time to reach thermal transport equilibrium.

Since the plasma is a good conductor, one must also consider the time for current penetration or equilibration of the current distribution. The simple estimate, as used in Table I, is the skin time based on the plasma size $a$ and the conductivity $\sigma$ as in Eq. (2.4).

This outline of the physics of hot, magnetically confined plasmas indicates the objectives of diagnostic techniques. Appropriate experimental methods must be found to measure all the physical variables well.

\section{EQUILIBRIUM MAGNETIC CONFIGURATION}

Because the time scale for ideal MHD processes is so short, the magnetic configuration is always a solution of the 
TABLE I. Characteristic lengths and times for tokamak plasmas.

\begin{tabular}{lccc}
\hline \hline Phenomenon & Quantity & Definition & Typical values \\
\hline Charge neutrality & Plasma frequency & $\omega_{p e}=\sqrt{\left(n e^{2} / \varepsilon_{0} m\right)}$ & $5 \times 10^{11} \mathrm{~s}^{-1}$ \\
Gyromotion & Debye length & $\lambda_{D}=v_{e \mathrm{th}} / \omega_{p e}$ & $10^{-5} \mathrm{~m}$ \\
& Gyrofrequency & $\Omega_{e}=e B / m$ & $5 \times 10^{11} \mathrm{~s}^{-1}$ \\
& Gyroradius & $\Omega_{i}=e B / M$ & $3 \times 10^{8} \mathrm{~s}^{-1}$ \\
& & $\rho_{e}=v_{e \mathrm{~h}} / \Omega_{e}$ & $10^{-5} \mathrm{~m}$ \\
Ideal equilibrium & Alfvén time & $\rho_{i}=v_{i \text { th }} / \Omega_{i}$ & $10^{-3} \mathrm{~m}$ \\
Electron equilibration & $e-e$ collision time & $\tau_{A}=\frac{R}{B} \sqrt{\left(\mu_{0} n M\right)}$ & $0.1 \mu \mathrm{s}$ \\
Ion equilibration & $i-i$ collision time & $\tau_{e e} \approx n \lambda_{D}^{3} / \omega_{p e}$ & $0.2 \mu \mathrm{s}$ \\
$T$ equilibration & $e-i$ collision time & $\tau_{i i}=\sqrt{M / m} \tau_{e e}\left(T_{i} \sim T_{e}\right)$ & $8 \mu \mathrm{s}$ \\
Drift instabilities & Drift frequency & $\tau_{e i} \approx \frac{M}{m} \tau_{e e}$ & $0.5 \mathrm{~ms}$ \\
Transport & Energy confinement & $\omega_{*} \approx v_{i} / L_{n}$ & $3 \times 10^{6} \mathrm{~s}{ }^{-1}$ \\
& Skin time & $\tau_{E}[\mathrm{Eq} .(2.9)]$ & $100 \mathrm{~ms}$ \\
& Density scale length & $\tau_{s}=\mu_{0} \sigma a^{2}$ & $1 \mathrm{~s}$ \\
\hline \hline
\end{tabular}

Grad-Shafranov equation [Eq. (2.3)]. However, because the boundary conditions (external coil currents, etc.) or internal state $[p(\psi)$ and $I(\psi)]$ may change with time on slower time scales, the magnetic equilibrium configuration may be time dependent. The diagnostic task is to determine the configuration as a function of time.

\section{A. Global properties}

The measurement of the basic global magnetic quantities is straightforward and mentioned here only for completeness. These quantities include the plasma current, loop voltage, and total energy content.

The plasma current is measured with a Rogowski coil, which is usually wrapped around the outside of the vacuum vessel like the poloidal loop (cf. Fig. 3). A Rogowski coil is a tightly wound helical coil that gives a direct measure of $\int B d l$ around the loop and hence the current within the loop. If the vacuum vessel can carry toroidal current, a correction for that must be made using the loop voltage and vessel resistance.

The loop voltage is measured with toroidal loop of the sort labeled $F$ in Fig. 3, which measures poloidal flux, albeit with respect to the major axis rather than the magnetic axis. In principle, any such loop is satisfactory for an equilibrium, because the loop voltage arises from a changing flux through the central region that is common to all the coils. In practice, a sum of coils, e.g., $F 1-F 4$ in Fig. 3(a), is used to obtain a result which is correct even if the plasma is moving. If the plasma current is time dependent, the loop voltage is somewhat ambiguous. It may be the voltage at the measuring loops, or it may be the voltage at the plasma surface, obtained by making inductive corrections. When the current or current distribution is changing, a more complicated analysis is required to obtain the $E_{\phi}(r, t)$ [cf. Sec. IV.D].

A useful measure of total energy content follows from Eqs. (2.1), which can be shown to require that $p+B^{2} / 2 \mu_{0}=$ constant. The plasma pressure is associated with a decrease in magnetic field and thus flux through a diamagnetic loop, e.g., the poloidal loop in Fig. 3. The measurement actually gives $\int p_{\perp} d V$ in principle, but because the plasma beta $\left(\beta \equiv 2 \mu_{0} p / B^{2}\right)$ is small in tokamaks, the diamagnetic contribution to the flux through the diamagnetic loop can be difficult to extract. Although this is a significant limitation for Ohmic discharges, the higher betas obtained with substantial auxiliary heating can be readily determined.

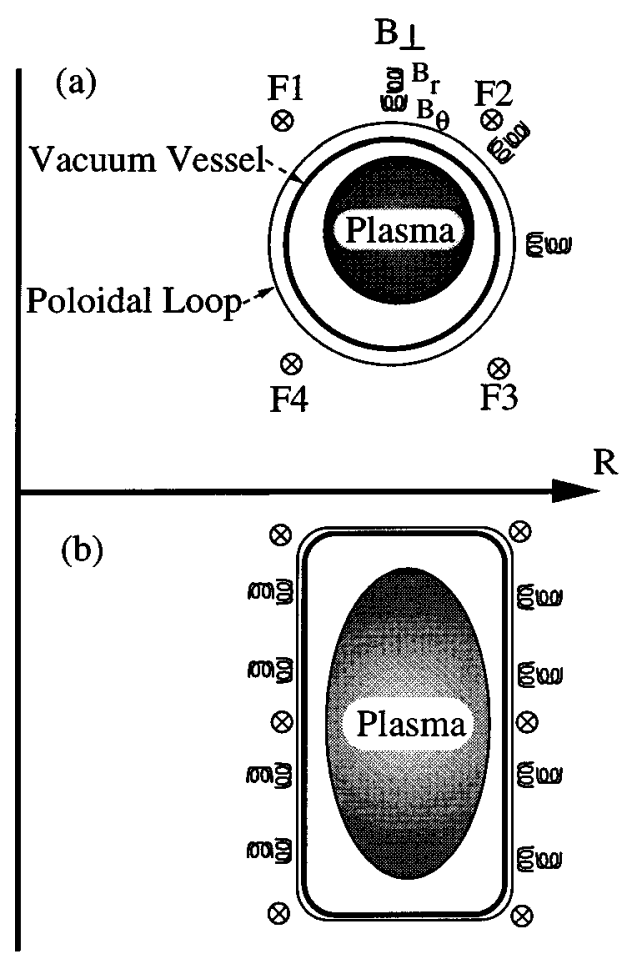

FIG. 3. Placement of magnetic diagnostics on circular and rectangular vacuum vessels. The poloidal loop measures toroidal magnetic flux. The $F$ loops run in the toroidal direction and measure poloidal flux. The $B_{r}$ and $B_{\theta}$ coils are small and oriented to measure the corresponding components of local field, the components perpendicular to the main toroidal field. 


\section{B. Circular cross sections}

Although the experimental determination of the magnetic configuration specified by the solution of the Grad-Shafranov equation (2.3) must be performed on all tokamaks, there is a major practical difference in technique between the simpler circular cross sections (i.e., Fig. 1, axis $A$ ), which include all the earlier tokamaks, and the more complex magnetic configurations, which include most modern tokamaks. The circular case is simple and is mentioned here only for completeness and for comparison with the noncircular case.

In the absence of specific coils to shape the plasma, the magnetic surfaces are approximately nested circles for modest aspect ratios $(R / a>3)$ and low beta. The magnetic axis is shifted outward from the center of the outermost flux surface by the Shafranov shift, which depends on the pressure and current distribution only through the parameter $\Lambda \equiv \beta_{p}+l_{i} / 2$, and is small compared with $a$. The magnetic configuration is thus effectively specified by only three parameters. These are usually chosen as the $R, z$ coordinates of the center of the outermost flux surface and the value of $\Lambda$. Since the three parameters determine the magnetic fields and thus the signals on poloidal flux loops ( $F$ in Fig. 3 ) and magnetic coils ( $B_{\perp}$ in Fig. 3 ), which measure the poloidal and radial components of $B_{\perp}$, a great variety of signal sets can be inverted to obtain the parameters. If circular loops can be placed concentric with the usual plasma axis, as illustrated by the poloidal loop in Fig. 3(a), special coils can be fabricated to determine the three parameters with great accuracy and minimal data analysis.

\section{Noncircular cross sections}

The noncircular case is much more difficult because the configuration can no longer be described by only a few parameters that have to be extracted from magnetic data. Instead, a great deal more data [suggested by the multiplicity of coils and loops in Fig. 3(b)] must be used to ascertain solutions which are mathematically underdetermined in a formal sense but which should be physically overdetermined in the sense of having some redundancy in the data. The problem is underdetermined in the sense that no finite set of observations determine a continuous boundary for the twodimensional equation. This aspect is usually resolved by the mathematically and physically reasonable approach of expanding in a set of basis functions and retaining only enough terms to represent necessary physical complexity. Configurations of coils and loops must be chosen to provide an accurate determination of all the terms in the expansion. More than a minimal set of coils is used with a fitting routine to provide improved accuracy and immunity from faults in one or more individual coils.

There is no standard approach to implementation for noncircular cross sections as there is for circular ones. The coil sets and algorithms have been developed for the particular features of each device. Several illustrative examples are detailed below, but certain general features should be noted. The analysis provides an accurate map of the flux surfaces; the position of the outermost flux surface or separatrix is determined with $0.01 \mathrm{~m}$ for plasmas of 0.5 to $1 \mathrm{~m}$ minor
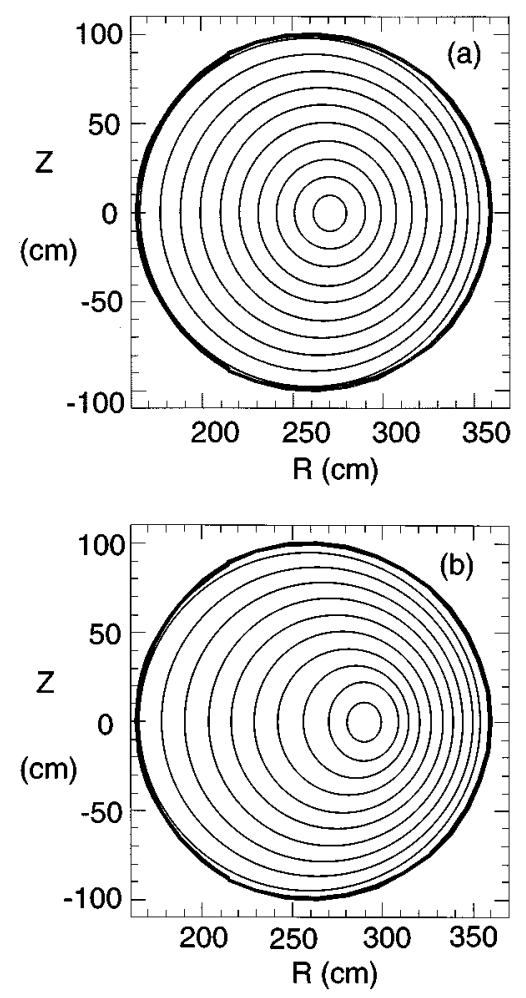

FIG. 4. Typical magnetic configurations in TFTR: (a) At low $\beta$, the flux surfaces are close to circular with a small Shafranov shift to the outside; (b) at higher $\beta$, the shift becomes much stronger and small deviations from circularity develop (Zarnstorff, 1995).

radius. The analysis is numerically complicated and computationally intensive; the full computation is performed at only a few times in each discharge, but this is not a serious limitation because the configuration changes, at most, slowly with time. Position information for feedback control purposes is obtained more simply and quickly. Unlike the circular case, for which the magnetic configuration depends only on the single plasma parameter $\Lambda$, the noncircular configuration does depend upon the current and pressure profiles of Eq. (2.3) independently. Conversely, these profiles can, in principle, be extracted solely from magnetic data, but the accuracy of the magnetic data is insufficient in practice. Instead, some information from other diagnostics, to be discussed later, is combined with the magnetic data for the computation.

A comparatively simple example is provided by TFTR, for which the flux surfaces are reasonably close to circular (Fig. 4). For more complicated cases, the flux surfaces must be calculated with suitable numerical codes, among which EFIT (Lao et al., 1985) is an important example. Typical results of this code are shown in Fig. 5(a) for DIII-D (at General Atomics, San Diego, CA) and in Fig. 5(b) for Alcator C-MOD (at MIT, Cambridge, MA) (Hutchinson et al., 1994).

\section{DETERMINING THE LOCAL FLUID VARIABLES}

\section{A. Density}

Contemporary plasma density measurement relies on the same physical principles that have been used for the past 30 


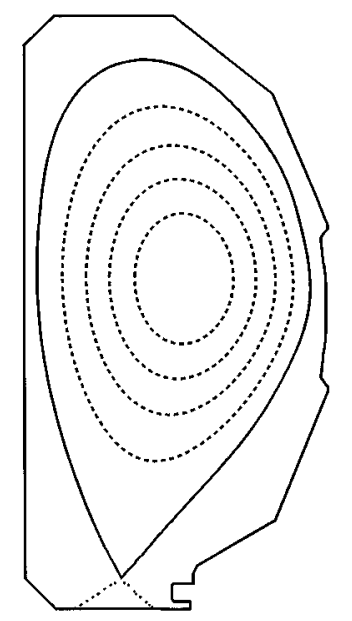

(a)

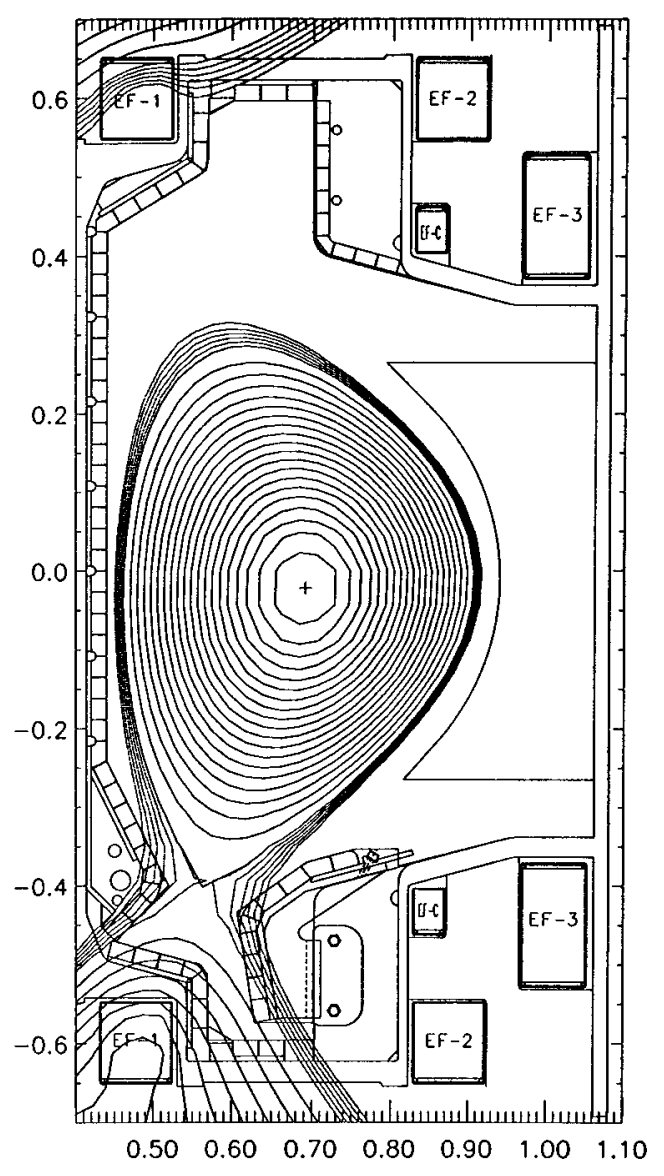

(b)

FIG. 5. Typical magnetic configurations calculated from EFIT: (a) DIII-D (Lao, 1995); (b) Alcator C-MOD (Hutchinson et al., 1994). Both are single null (at the bottom) divertor configurations.

years, but improvements in implementation have produced qualitatively better results. The principal technique is based on the fact that the index of refraction for electromagnetic waves can be written as $n=\sqrt{1-\omega_{p e}^{2} / \omega^{2}}$ for $\omega>\omega_{p e}$, which is true even in the presence of magnetic fields provided that the wave electric field is polarized along $B$. Provided $\omega \gg \omega_{p e}$, the radical may be expanded to imply a change in refractive index linearly proportional to plasma density. An interferometer viewing through the plasma observes a phase shift proportional to $\int n_{e} d l$ along the path, with a simple and precise calibration factor.

The application requires a subtle compromise. If the interferometer frequency is chosen too high, the phase shift will be small and accuracy therefore poor. If the frequency is too low, however, sight lines not through the magnetic axis will have a significant component of $\nabla n_{e}$ perpendicular to the direction of propagation, leading to refraction. Significant refraction can, at worst, deflect the beam from the detector and, at best, render interpretation quite difficult. Modern interferometers result from the development of far-infrared technology to produce systems operating at wavelengths of hundreds of microns, chosen so that refraction is not important, and the approximation $\omega \gg \omega_{p e}$ is well satisfied. The systems must then be sufficiently stable that phase shifts of 0.1 radian or less are measurable.

High-accuracy interferometers are necessary because the direct observations are those of line-integrated density, which must be inverted to find local densities. This is a problem common to many plasma diagnostics, not to mention general tomographic applications. However, general tomographic procedures are rarely applicable to tokamak plasmas. On the one hand, tokamak geometry precludes obtaining the complete set of views required for full tomography; but on the other hand, the symmetry and plasma properties simplify the problem. Special approaches have been developed for plasmas. First, the density is generally assumed to be constant on flux surfaces, $n(\psi)$. [In a few cases, one additional term, $n_{1}(\psi) \cos \theta$, is included to permit the lowest-order fluxsurface variation that often appears theoretically, but no additional terms are customarily included for equilibria. More elaborate systems have been proposed.]

The optimal spatial resolution in the $n(\psi)$ profile is determined by the number of channels $N$ in the interferometer, according to a sort of Nyquist sampling theorem. For evenly spaced channels, only wavelengths longer than $2 a / N$ are measurable in the density profile. Increasing the number of channels to improve spatial resolution is subject to two important limitations. First, the interferometer beams have diffraction-limited sizes characteristic of their wavelength. Beams separated by less than the beamwidth would not contribute independent information. Technical limitations often restrict the number of channels even more severely. Second, a channel only contributes new information if its precision is greater than the difference between adjacent signals. Roughly speaking, if the difference between signals in adjacent channels is less than the noise or random measuring error in the channel, the channel contributes little information. This is reasonably correct for a collection of point measurements but is especially true for inversions, which are quite sensitive to errors in data.

The time resolution is determined only by electronics, most usually in the technique for measuring phase in the interferometer. It is always much faster than the transport time scale and is customarily in the range $1-10 \mu \mathrm{s}$.

Several inversion techniques are currently used. If only a few channels are available $(N<5)$, or even if there are more 
channels but a simple parametrization is desired, a simple functional form for $n(r)$ with only a few adjustable parameters, for example,

$$
n(r)=n_{0}\left[1-(r / a)^{\alpha}\right]^{\beta} \text { or } n(r)=n_{0} \exp \left[-(r / b)^{\alpha}\right]
$$

with $n_{0}, \alpha$, and $b$ or $\beta$ free, may be fit to the data. In principle, an error analysis could be performed to determine if the resulting fit is adequate, given the errors in the lineintegrated data, but this is rarely presented. However, profiles obtained with more channels and more general analysis, as shown below, generally show rather smooth, monotonic forms consistent with Eq. (4.1).

If the flux surfaces are circular, the conventional Abel inversion may be used:

$$
n(r)=\frac{-1}{\pi} \int_{r}^{a} d r^{\prime} \frac{\left(d N / d r^{\prime}\right)}{\sqrt{r^{\prime 2}-r^{2}}} .
$$

The continuous function $N(r)$ describing the line-integrated data is obtained by fitting splines to the discrete set of experimental points. The process requires some care. Even small errors in adjacent channels can produce large oscillations in $d N / d r^{\prime}$, causing the $n(r)$ from Eq. (4.2) to be unphysical. By choosing coarser splines, an approximate but smoother fit to the data points can be found which gives a reasonable $n(r)$, but with lower spatial resolution than might be inferred from the number of channels.

Other approaches, which are necessary if the flux surfaces are noncircular, include dividing the plasma into a discrete set of finite annular regions, bounded by flux surfaces, and assigning an unknown constant density to each region. The constants are then adjusted for a best fit to the interferometer data. The result is usually presented as a smooth curve fit to the values. A useful extension of this technique is provided by Park (1989). Even if $n(\psi)$ is computed, the result is often displayed as a function of a physical position.

Alternatively, $n(\psi)$ may be expanded as a finite sum of basis functions whose coefficients are adjusted to fit the observations. Various choices are possible, for example, the Zernicke polynomials used by Cormack (1964). However, none is particularly natural in the sense of providing functions that resemble typical density profiles. This approach has been used for the inversion of other chordal data, e.g., X-ray emission by Granetz and Smeulders (1988), as well as density (Howard et al., 1988).

A typical system is that on TFTR (Mansfield et al., 1987) as diagrammed in Fig. 6. It provides ten channels vertically through the plasma. Representative profiles, used for transport analysis (Efthimion et al., 1991), are shown in Fig. 7. Typical of the best results obtainable from interferometry is the profile of Fig. 8, comprising 20 channels spaced across the profile that are shifted slightly from shot to shot to interpolate the dense coverage shown (Brower et al., 1992). Alternatively, such systems can provide 20-channel resolution on single shots.

In all cases, modern interferometer systems provide density profiles with spatial resolution better than $\sim 20 \%$ of the radial dimension and virtually continuous time histories. There is no indication that they are missing significant struc-

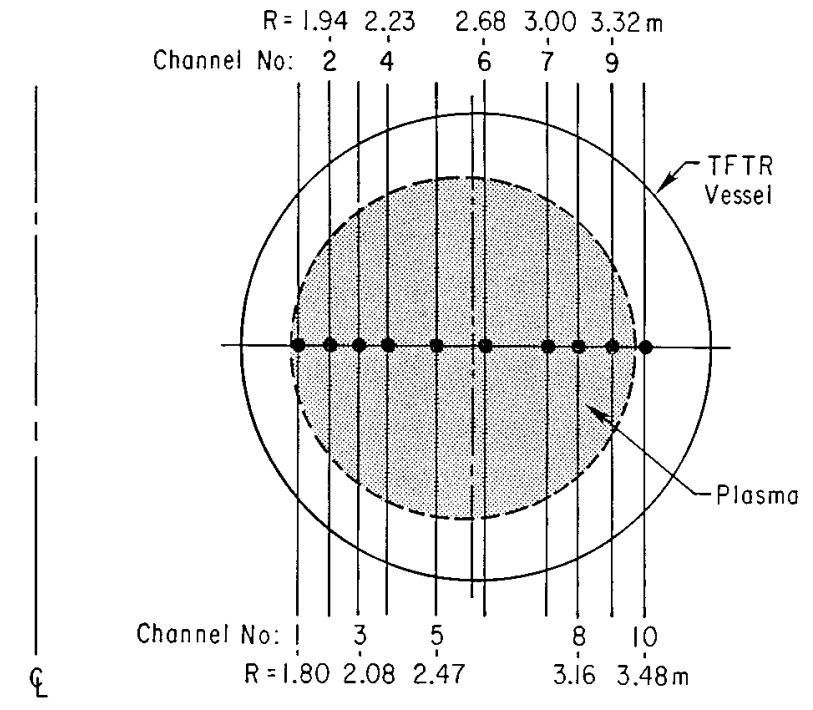

FIG. 6. Views of the ten-channel far-infrared interferometer on TFTR. The sight lines range over both sides of the magnetic axis (Efthimion, 1995).

tural features, although there could be some small asymmetries and effects associated with rational surfaces that escape detection.

Beyond the intrinsic difficulties associated with chordintegrated data, there is one important systematic limitation on the diagnostic technique. The spatial resolution is constrained by the diffraction limit on the beam size. Under some circumstances-the H-mode is one example (see the discussion accompanying Fig. 21) - the plasma develops very steep density gradients. Scale lengths of less than a centimeter cannot be resolved with interferometry. Other techniques are required.

The second important source of density information is a by-product of Thomson-scattering temperature measurements, discussed in Sec. IV.B. Elementary scattering theory

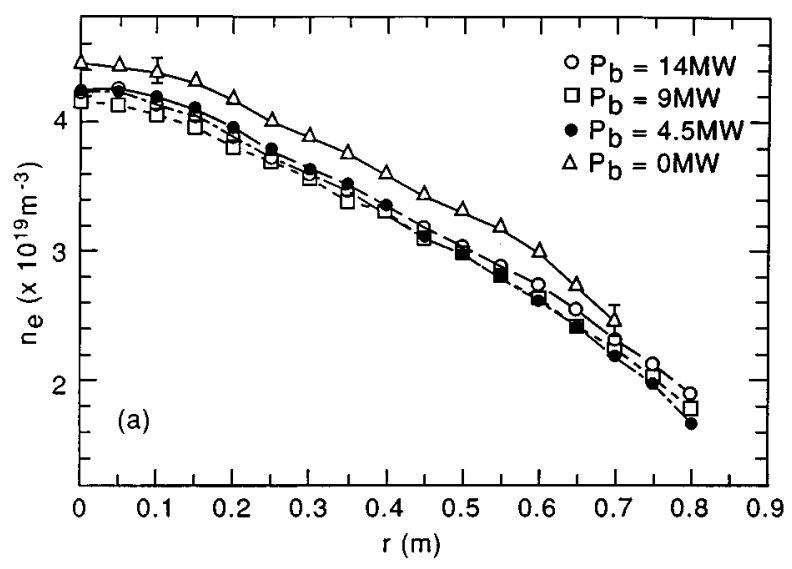

FIG. 7. Typical density profiles from TFTR. The profiles remain nearly constant in this case as the power in the neutral heating beams is increased (Efthimion et al., 1991). (The symbols distinguish the curves for different conditions; they do not indicate independently measured values of density.) 

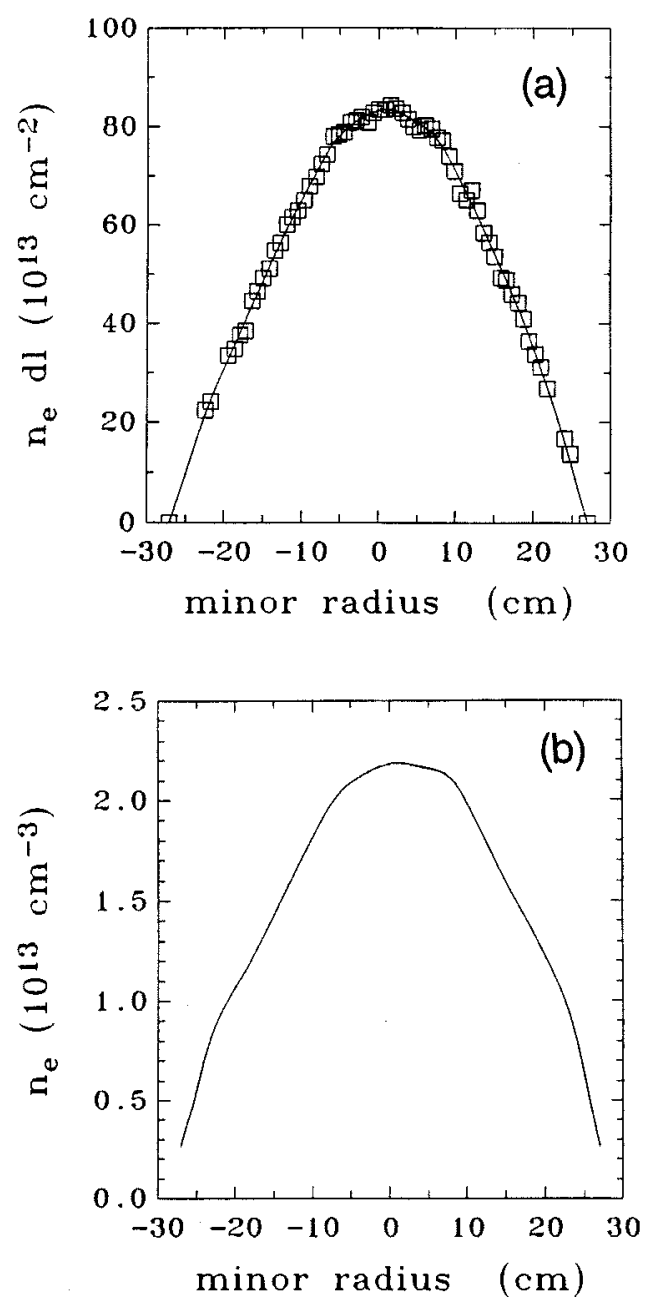

FIG. 8. Data from a large number of interferometer chords on TEXT [shown by boxes in (a)] and the inversion to obtain local density (b). The limiter for this case is at $28 \mathrm{~cm}$ (Brower et al., 1992).

implies that the intensity of incoherent scattered light is proportional to the number of scatterers. A measurement of the absolute intensity of the scattered light therefore determines the density. The natural temporal and spatial resolutions of Thomson scattering are excellent; the limitation is accuracy. Thomson-scattering systems are generally close to noise limits, and absolute intensity measurements are further prone to systematic error. The systematic error can be greatly reduced by extracting only a density profile shape from the Thomson data and adjusting the multiplicative constant to match the interferometer data. In this combination, the Thomson profile is a valuable complement because it gives shape information independent of an inversion process; e.g., it confirms the absence of small-scale, large amplitude density variations. Illustrative data will be shown in Sec. IV.B with other Thomson-scattering results. Another limitation on Thomson scattering as a density diagnostic is that it provides data only at one or a few times during a discharge. Continuous time evolution cannot be followed.

A third source of density information comes from reflectometry, the complementary consequence of $n=\sqrt{1-\omega_{p e}^{2} / \omega^{2}}$. An electromagnetic wave can propagate where $\omega>\omega_{p e}$; but if it propagates into a region of increasing density, it will be reflected from the layer $\omega=\omega_{p e}$. Using various sorts of interferometric techniques, information on $n(r, t)$ can be extracted. The technique is especially valuable and sensitive for measuring an $r_{n}(t)$, the position of a particular value of density as a function of time, and it is quite useful in regions of steep density gradient; but it fails near the center and in other regions where $d n / d r$ is small. The technique is included in the review by Stott (1992); a good example of its application is found in Bartlett et al. (1990).

\section{B. Electron temperature}

Two valuable techniques for measuring electron temperature are available, the benchmark technique of Thomson scattering and the versatile technique of electron cyclotron emission (ECE). The principle of Thomson scattering is well established (e.g., Sheffield, 1975), being simply the Doppler frequency shift in the light scattered from moving free electrons. A viewing spectrometer observes the light scattered nearly perpendicular to the incident laser beam, the tight collimation of the crossed laser beam and viewing optics providing spatial resolution of order one centimeter. The recent advances have been in technique: improved accuracy, detector systems to provide a complete spatial profile on each laser pulse, and multipulse lasers to provide temperatures at several times during a single tokamak discharge. The first two have been especially important in providing $T_{e}(r)$ as necessary for transport analysis.

Although specifics of technique are generally beyond the scope of this article, some aspects of Thomson scattering deserve discussion. Because transport analysis requires the spatial derivative $d T_{e} / d r$, instantaneous full profiles to avoid shot-to-shot variations are important, not merely convenient for efficiency. The first steps in this direction were obtained by simply duplicating the single-point spectrometer systems to give multiple points. Further progress came with the use of two-dimensional detectors with many pixels that could be coupled to imaging spectrometers, providing nearly continuous coverage in wavelength and position along the laser beam. Perhaps the most advanced of such systems is that on TFTR (Johnson et al., 1985). An example of profiles from this instrument is shown in Fig. 9. Another example of modern Thomson-scattering results is shown in Fig. 10 from DIII-D; this is an excellent example of the high spatial resolution and steep gradients that can be found. Figure 10(b) also illustrates another technological development, the use of several multipulse laser systems to provide time histories of temperature and density through a discharge. The steep edge gradients are typical of the tokamak H-mode (cf. Fig. 21 and discussion there).

An alternative implementation of Thomson scattering is termed LIDAR, which relies on the time of flight of the laser pulse through the plasma to provide spatial resolution. This principle has been used in JET (Salzmann et al., 1988). The light scattered into a small cone close to the backward direction is collected and analyzed as a function of time as a very short laser pulse propagates through the plasma. The approach is attractive for technical reasons: a single aperture view of the plasma provides data along the entire line of 

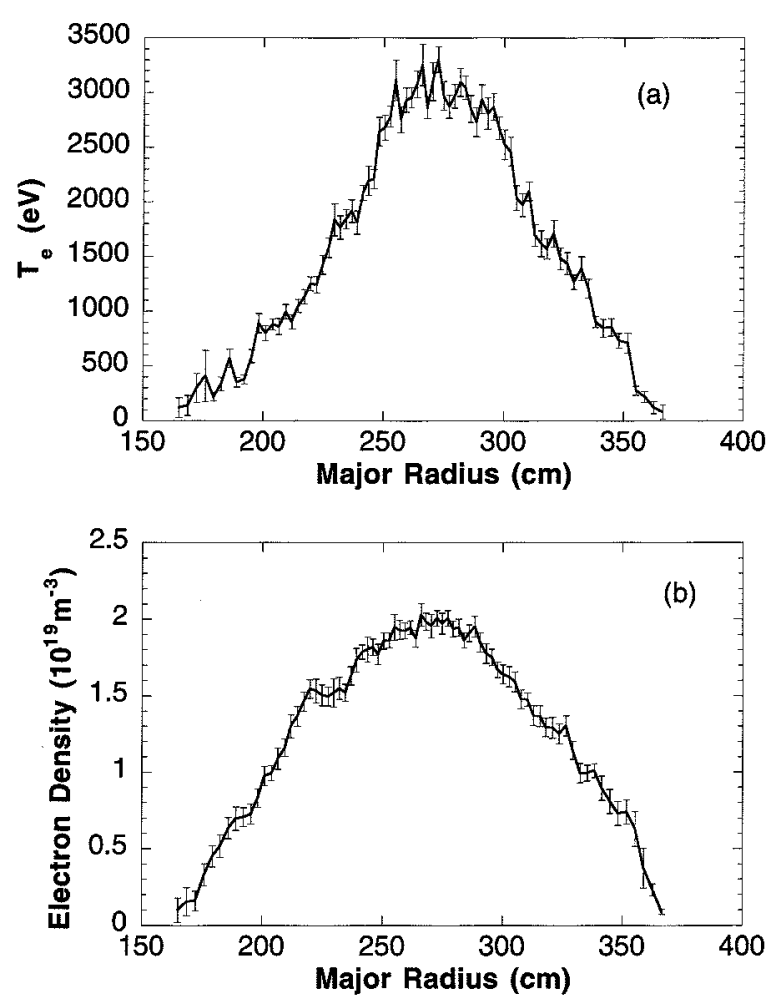

FIG. 9. Representative temperature (a) and density (b) profiles from the TFTR Thomson-scattering system for a standard 2 MA discharge with no additional heating. The magnetic configuration is close to that in Fig. 4(a). In addition to the statistical error bars shown, there is a $5 \%$ systematic uncertainty in the electron temperature. These systems provide a large number of independent radial points at a single time (Grek, 1995).

sight. (A typical profile is shown below as Fig. 12.) Limits on the speed of data acquisition and the shortness of a laser pulse with sufficient energy to provide adequate scattered signal restrict the spatial resolution to larger values, $\sim 10 \mathrm{~cm}$, that are sufficient only for larger devices.

Modern Thomson-scattering systems provide $T_{e}(r)$ with a spatial resolution at least $a / 10$ and an accuracy of $10 \%$, usually better. They also provide density profiles. Their principal limitation is that they give only a snapshot in time. Even the most sophisticated multilaser, multipulse systems are limited in the choice of time histories that can be measured. The most important limitation follows from the small size of the Thomson cross section: even with powerful lasers, photon statistics impose error bars of at least a few percent. Therefore small effects cannot be discerned, independent of the spatial or temporal resolution of the system. ECE techniques have been developed to provide continuous time coverage and finer temperature resolution; they often offer operational advantages of technical simplicity and immediately interpretable data as well.

The ECE measurement of $T_{e}$ relies on the blackbody optical opacity of the plasma at the electron cyclotron frequency, the second harmonic, and often higher harmonics. Only near the edge in smaller machines is the optical depth sometimes insufficient for good measurements. The fundamental principles are outlined in Bekefi (1966), and a more recent review is provided by Bornatici et al. (1983). The technique is particularly effective because the plasma is opaque only at the cyclotron frequency. It becomes transparent again for higher and lower frequencies. (Implicit for transparency is the condition that $\Omega_{e}>\omega_{p e}$, but this generally obtains in tokamaks.) Since the magnitude of the magnetic field, and therefore the cyclotron frequency, varies primarily as $1 / R$, the measured radiation intensity $I(\omega)$ can be mapped directly to $T_{e}(R)$ along the line of sight of the radiometer. Figure 11 illustrates the principle. The naturally high temperatures of a tokamak imply strong blackbody radiation levels, but the typical magnetic fields place the radiation in the far infrared where detection is the most difficult. These technical problems have been the focus of the development effort and determine the quality of data obtainable from ECE. The frequency resolution may be obtained by either optical or electronic techniques. The latter technique uses a mixer and conventional electronic filtering; it offers the high sensitivity of a superheterodyne receiver, but requires special care for absolute intensity calibration. Two optical techniques are common. One uses a grating spectrometer and an array of detectors, the number of which determines the spatial resolution. Like the electronic technique, this offers a continuous $T_{e}(t)$ from each detector, with a time resolution limited only by the amount of filtering required to obtain a good signal-to-noise ratio from the detector. Absolute calibration requires care, and relative detector calibration must be especially accurate to infer temperature gradients. The second optical method avoids this problem by ingeniously using a single detector in a swept Michelson interferometer. Calibration is simpler, but at the loss of continuous time information. A $T_{e}(r)$ profile is obtained only over the sweep time of the interferometer. The consistency and accuracy among the various techniques for measuring $T_{e}$ are illustrated in Fig. 12, which compares Thomson scattering with two ECE systems on JET.

Assuming adequate signal levels in the detector, we see that there remain two important factors in the use of ECE data. One is the difficulty in calibration to obtain and maintain absolute temperature measurements. Thomson scattering is often used as an alternative to absolute blackbody sources, but some care is required in complex geometries to determine which points from each diagnostic are on the same flux surface, and temperature constancy on flux surfaces is assumed. Different chords through the plasma are always used. A more interesting factor concerns the nature of the measurement. By its nature, Thomson scattering measures the velocity distribution of the bulk of the electrons. On the other hand, ECE emission is blackbody only if the electron distribution is Maxwellian. Significant deviations from Maxwellian, as can arise from Ohmic heating at low density, ECH, or lower hybrid current drive, can cause large anomalies at the various harmonics. These can even be used for diagnostic purposes to obtain some properties of the distribution function. Care in the interpretation of ECE data is required in circumstances where non-Maxwellian distributions may arise. These technical aspects and subtleties of interpretation are discussed by Costley (1991a). Nevertheless, ECE has proved invaluable in supplying high-resolution $T_{e}$ profiles and time evolution. It can even measure the temperature 

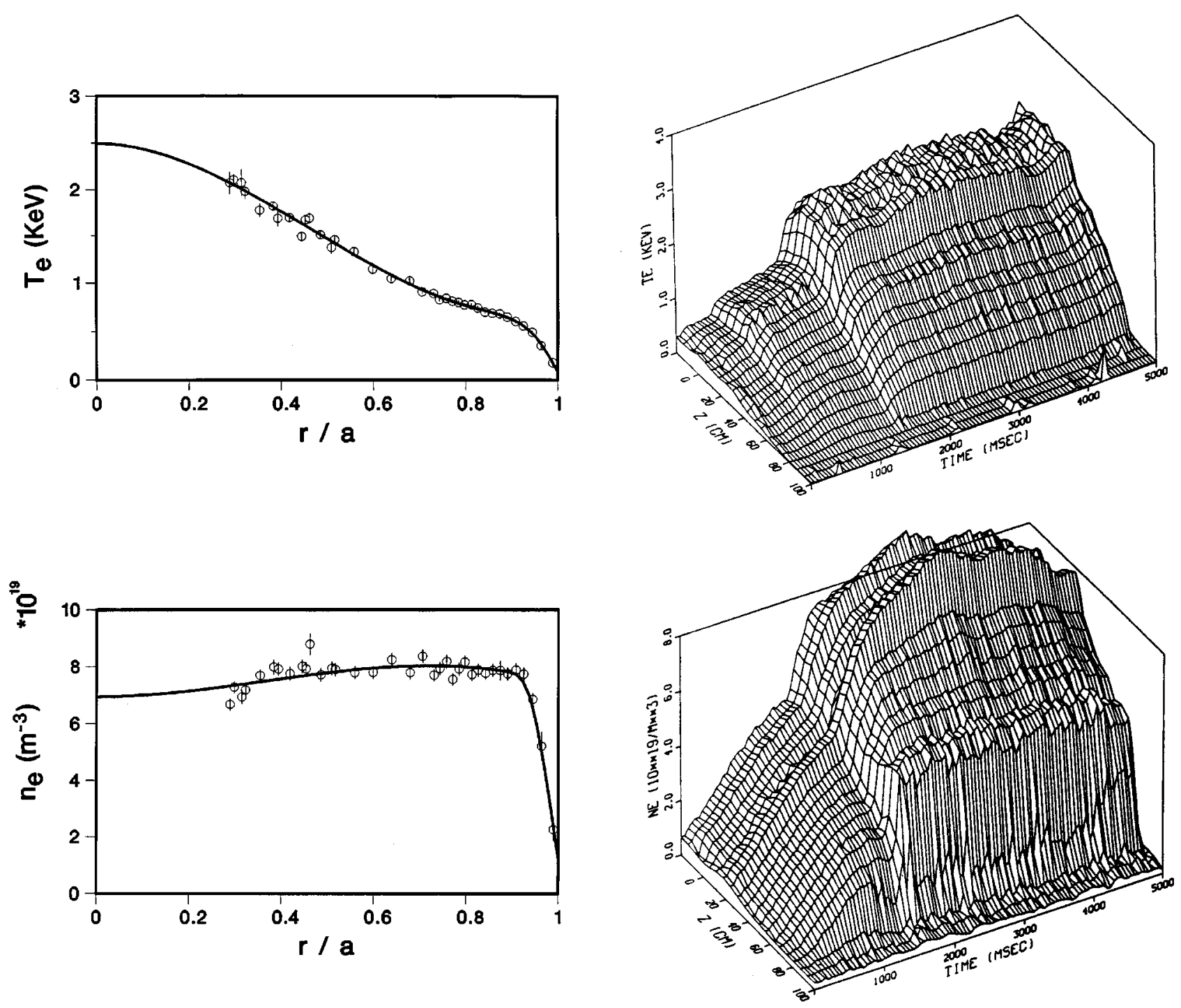

(a)

(b)

FIG. 10. Representative temperature and density profiles from the DIII-D Thomson scattering: (a) Conditions are similar to those of Fig. 21. Note especially the very sharp gradient in density at the edge resolved by the system. (b) Time development of the profiles for similar conditions using multipulse lasers. Transport analysis and the profiles of (a) apply to the steady conditions like those between 3.0 and 4.0 seconds (Lao, 1995).

fluctuations associated with large-scale modes (cf. Sec. VI.B: sawteeth and $m=1,2, \ldots$ modes) and turbulence (cf. Sec. VI.B).

\section{Ion temperature}

Two principal techniques are employed to measure ion temperatures. Hydrogen ion temperatures are determined from the energy spectrum of charge-exchange neutrals emerging from the plasma. (A neutral atom entering the plasma has roughly equal probability of being ionized or charge exchanging with a plasma ion. The new neutral, representative of the plasma ion distribution, may escape the plasma and be detected-subjected to a second charge exchange and energy analyzed. The detector views along some line of sight through the plasma, and the measured spectrum depends on a complicated integral of the neutral density, ion density, and ion temperature along the sight line.) A good illustration of charge-exchange results is provided by Fig. 13 (Roquemore et al., 1985), which shows the distribution function of the main deuterium ions as well as the distribution of a small minority of hydrogen ions heated to a higher temperature by ICRH.

The analysis of neutral particle spectra naturally determines the full distribution function $f(v)$, not merely the ion temperature, and measurable signal extends well into the tail, at least to several times the thermal velocity and often much higher. [This contrasts with many spectroscopic Doppler techniques, including Thomson scattering and impurity ion temperature measurements, for which the line shape, in prin- 


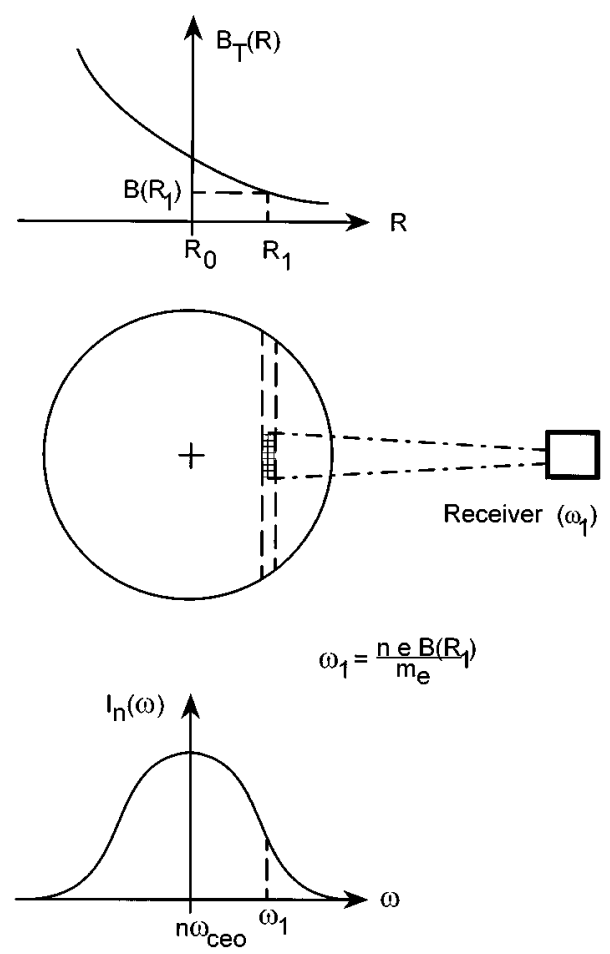

FIG. 11. Operating principle of ECE temperature diagnostics. The spatial variation of the magnetic field and the restriction of blackbody radiation to a narrow band near the electron cyclotron frequency associate the radiation intensity at each frequency with the temperature at a particular position within the plasma. (A narrow antenna pattern for the receiver is also required.) The measurement is usually made at the fundamental $(n=1)$ or first harmonic $(n$ $=2$ ).

ciple, represents $f(v)$ but in practice must be fit to a Maxwellian with only an adjustable temperature and, possibly, drift velocity.] This capability is quite important because the lower collision frequency and larger orbit size of ions cause larger deviations from Maxwellian distributions. These are greatly exacerbated when neutral beams or ions cyclotron resonance heating (ICRH) is used, for both are copious sources of superthermal ions. Knowledge of the full distribution function is much more important for hydrogen ions than for electrons or most impurities. The ion distribution functions provide not only $T_{i}$ for transport analysis, but also the basic information necessary to analyze the kinetic effects associated with the heating processes, a rich area of physics.

There are some significant subtleties associated with the measurement of $f(v)$. The vector $\mathbf{v}$ must lie along the path to the detector. For example, if the sight line is approximately perpendicular to $\mathbf{B}$, easily realized in practice, one will measure $f\left(v_{\perp}, v_{\|}=0\right)$, which constitutes deeply trapped ions and introduces other possible problems. Regardless of configuration, one will obtain an $f(v)$, but never the full $f\left(v_{\perp}, v_{\|}\right)$. Measurements on strongly non-Maxwellian distributions are incomplete. In fact, the detail provided by charge-exchange spectra can sometimes prove a disadvantage: it can be difficult to extract a representative $T_{i}$ from the data.

The second technique for determining ion temperature measures the Doppler width of various spectral lines of plasma impurities. Although of secondary intrinsic interest,
ECE-LIDAR Temperature profile comparison

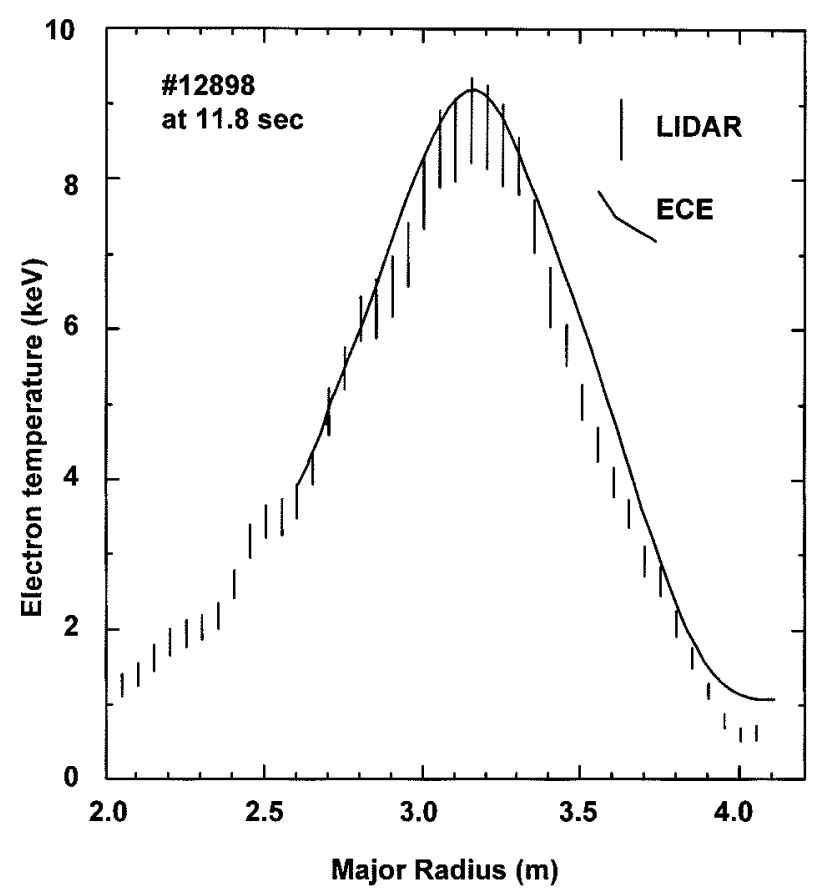

FIG. 12. JET $T_{e}$ ECE and LIDAR temperature profiles (Bartlett, 1995).

the impurity ion temperature reflects, with some calculable differences, the temperature of the dominant ions and is often easier to ascertain. As with many other diagnostics, the principles are well established and unchanged, but the application has greatly improved. In its basic form, the method offers limited spatial resolution because the ionization state giving rise to each line exists over a region within the plasma. The higher ionization states are restricted to the center, and low states to the edge; but the localization is not sharp. Depending upon plasma conditions, there may not be suitable impurity lines across the whole profile. However, the comparative simplicity of spectroscopic techniques in application and analysis made technical development attractive.

The major advance has been the introduction of neutral beams to improve spatial resolution and strengthen signals. Both heating beams (e.g., Isler et al., 1981 and Groebner et al., 1983), to be found on all large tokamaks, and beams specifically for diagnostic purposes (e.g., Fonck et al., 1984) have been used. Among other reactions, the injected neutrals undergo charge-exchange reactions with impurity ions, including the fully stripped species like carbon and oxygen which otherwise emit no line radiation, producing a cascade of lines assignable to the intersection of the neutral beam and spectrometer sight line. Since carbon and oxygen impurities are ubiquitous in tokamaks, suitable lines can be found everywhere. The configuration on TFTR is representative and shown in Fig. 14 (Synakowski et al., 1993). The crossed sight paths provide resolution $\Delta \rho<0.04$.

The analysis of this charge-exchange recombination radiation, which has been assigned different acronyms on different tokamaks, can provide excellent ion temperature profiles. A good example is shown in Fig. 15 from the DIII-D toka- 


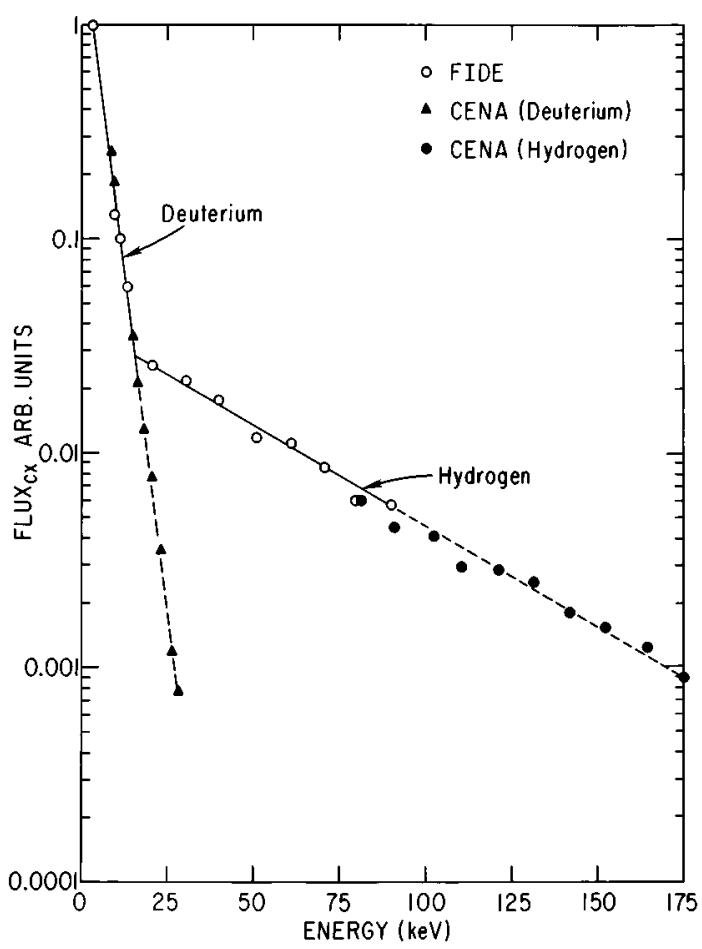

FIG. 13. Ion distribution functions in TFTR from charge-exchange neutral analysis (CENA). The plasma was heated with electromagnetic waves that preferentially heated hydrogen ions. The measurements clearly distinguished the different temperatures of the hydrogen and deuterium ions (Roquemore et al., 1985). (FIDE is the fast-ion diagnostic experiment.)

mak using the system described by Gohil et al. (1990).

In plasmas with deuterium and sufficient ion energies, neutron emission may also be used as an ion diagnostic. This development is important because future devices will produce even stronger neutron emission, and some present techniques will become more difficult, if not impossible. How-

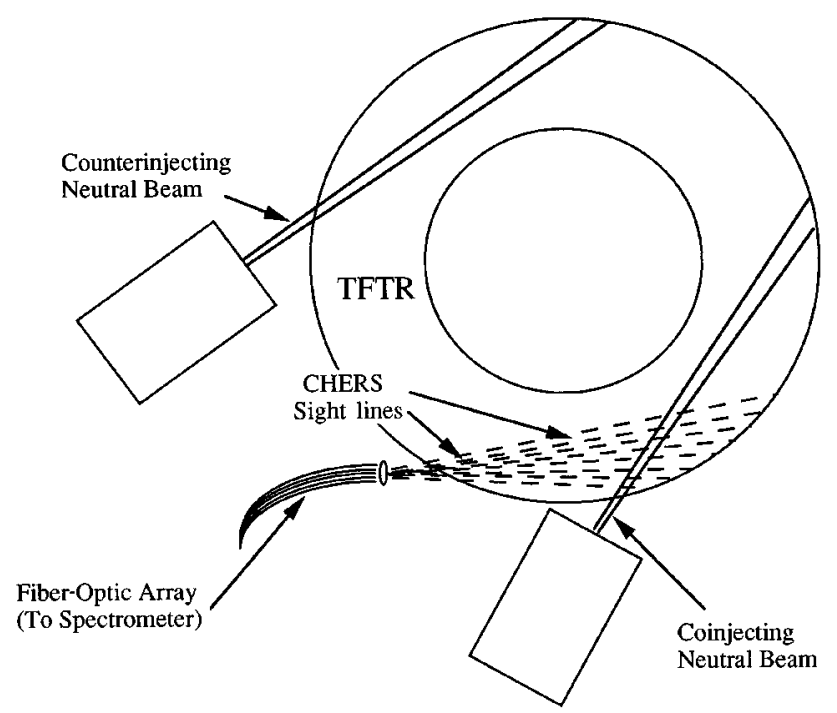

FIG. 14. CHERS (charge-exchange recombination spectroscopy) configuration on TFTR, illustrating the crossed sight lines that determine the spatial resolution (Synakowski et al., 1993).

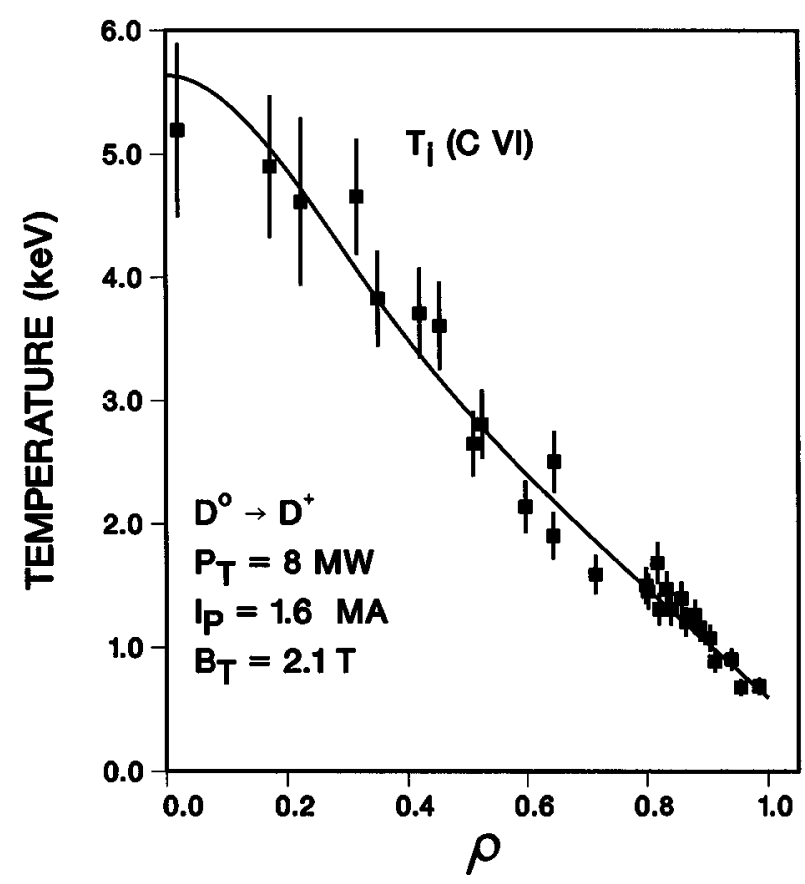

FIG. 15. Ion temperature profile in DIII-D from charge-exchange recombination spectroscopy for a very well-confined discharge condition (Groebner, 1994).

ever, the principal work so far has been establishing the methods and corroborating other measurements and codes (e.g., Strachan et al., 1994) rather than supplying a primary source of ion temperature data and therefore will not be discussed.

\section{Velocity}

Fluid velocity is actually a very subtle concept because of the complex composition of the plasma and the strong gradients. The obvious Eulerian velocity $\langle v\rangle=\int v f(v) d v$ differs from a Lagrangian velocity obtained by following an average particle, which is a guiding center or bulk fluid velocity. The difference is the diamagnetic drift velocity that develops in the presence of a pressure gradient:

$$
v_{d \theta}=\frac{\hat{b} \times \nabla p}{n e B} .
$$

The plasma is confined in the radial (normal to flux surface) direction. Fluxes in that direction are on a slow transport time scale, but substantial flow velocities on the magnetic surface are possible. The theoretical description is naturally in terms of velocity components parallel and perpendicular to the magnetic-field lines, but measurements are usually referred to laboratory coordinates $\phi$ and $\theta$, or more generally as $\phi$ and the direction on the flux surface perpendicular to the toroidal direction $\phi$. Regardless, the flow velocities constitute rotations. Several techniques can provide information on rotations.

Direct measurement of the Eulerian velocity of impurity species can be obtained from Doppler shifts of emission lines. With appropriate optics, both $v_{\phi}$ and $v_{\theta}$ can be measured. As with ion temperatures, the best spatial resolution results from charge-exchange recombination lines in crossed- 


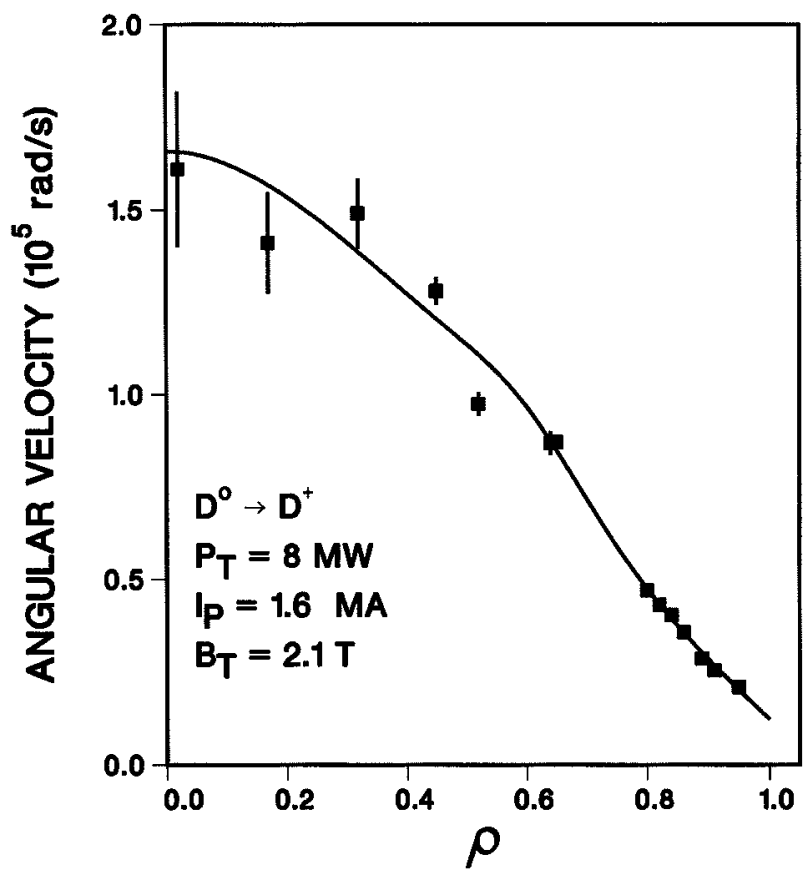

FIG. 16. Profile of toroidal rotation velocity in DIII-D for the same conditions as Fig. 15 (Groebner, 1994).

beam configurations. Figure 16 shows an example of the toroidal velocity profile in DIII-D obtained by this technique for a plasma that received a large toroidal momentum input from the heating beams.

Impurity velocities are measured in this process, whereas hydrogen ion velocities are usually desired. In the toroidal direction, there are no diamagnetic effects, and collisions are sufficient that all ions move with a common velocity. The subtleties arise for $v_{\theta}$, which requires more extensive analyses of the various species.

An alternative approach that determines the bulk fluid velocity is to measure the radial component of the electric field $E_{r}$ in the plasma. Since the plasma is an excellent conductor, the electric field in its rest frame must be zero: $E_{r}=v_{\perp} \times B$ in the most simple one-fluid analysis. This electric field is difficult to measure, but the plasma potential profile can be determined by one technique, a heavy-ion-beam probe (HIBP). A beam of massive ions, typically cesium or thallium, is accelerated to sufficient energy that the gyroradius exceeds the plasma size. Ions that ionize again in the plasma proceed on more curved trajectories and may be detected, the geometry of the detector fixing the position along the primary beam at which the ionization occurred. Since the charge was different entering and leaving the plasma, the ions suffer an energy change $\Delta U=e \phi_{p}$, giving the potential at the ionization point. Since the plasma potentials are typically $1 \mathrm{kV}$ and beam energies up to $2 \mathrm{MeV}$ may be necessary to traverse the plasma, the implementation is technically demanding. However, the interpretation of the result as rotation is clear. A comprehensive treatment of HIBP principles and results, as well as an extensive bibliography, may be found in Crowley (1994). A HIBP system installed on the TEXT (at University of Texas, Austin, TX) tokamak is shown in Fig. 17. An example of the potential profile for an Ohmic discharge is shown in Fig. 18. For this case, the potential is consistent with neoclassical theory and the ion pressure profile.

Useful supplementary information on rotation can also be obtained from other observations. The most important of these is the MHD activity that is nearly ubiquitous in tokamaks. These are presumed to be resistive tearing modes and include the $m=1$ modes often associated with sawteeth (cf. Sec. II.B) and $m=2,3$, or 4 detected with magnetic coils outside the plasma. In linear theory, these modes are purely growing; in experiments, the $m \geqslant 2$ modes saturate nonlinearly at small amplitude under most conditions. In any case, the mode structure should be stationary in the bulk plasma frame, and they are observed as oscillations in time only because the plasma rotates. In fact, the modes are resonant on integer $q$ surfaces and have helical structure for the perturbed quantities:

$$
\tilde{B} \propto e^{i(m \theta-n \phi)} .
$$

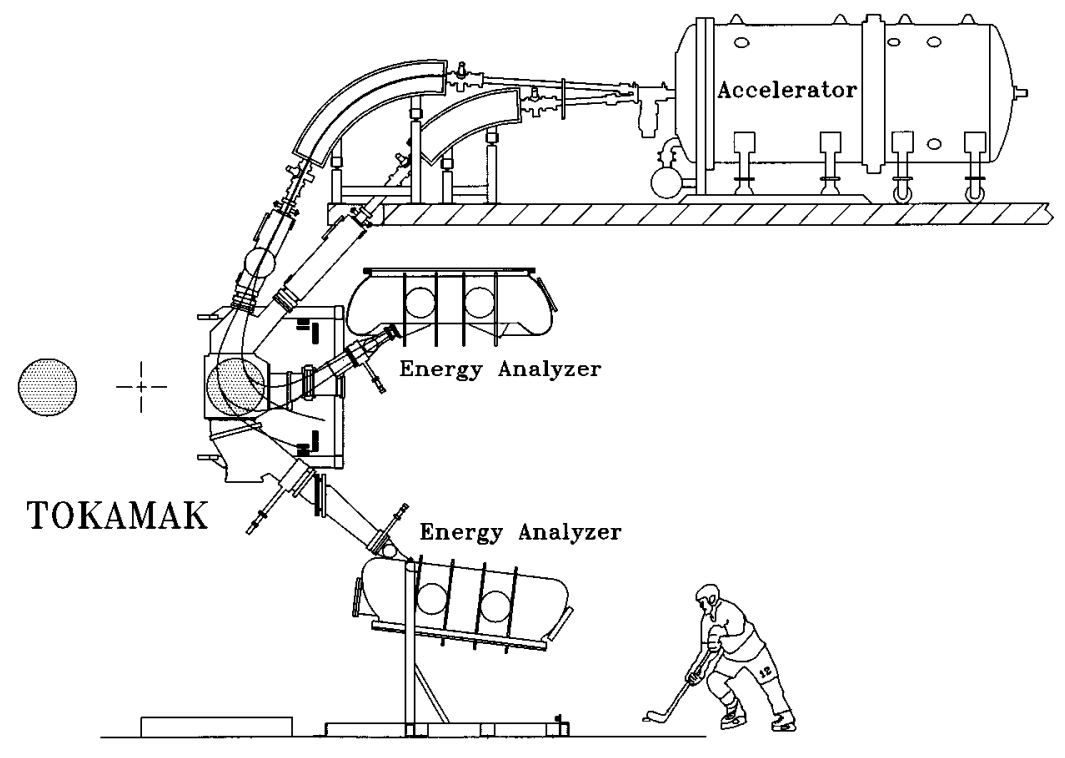

FIG. 17. HIBP schematic showing the system installed on TEXT. The beam trajectories must be adjusted to provide signal from the position of interest within the plasma (Schock, 1995). 


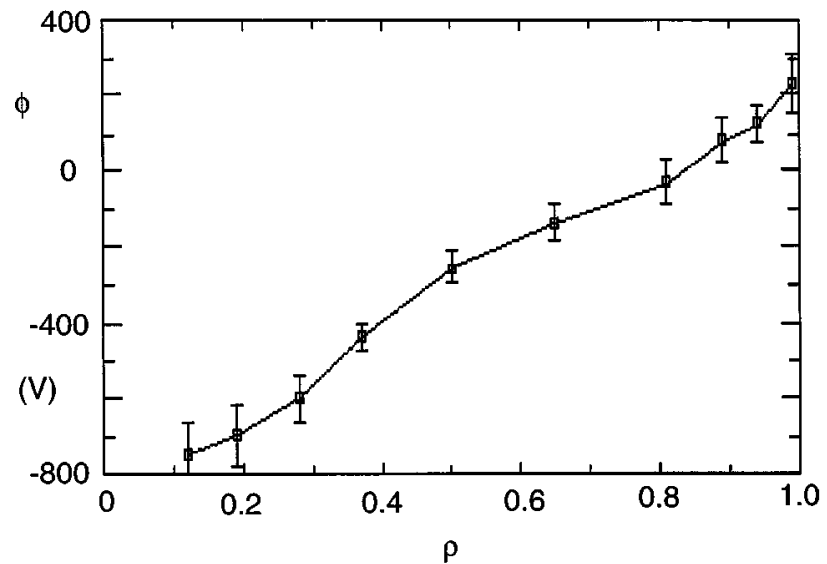

FIG. 18. Plasma potential profile from the HIBP system on TEXT. The solid line is a spline fit (Schock, 1995).

Rotation in either $\theta$ or $\phi$ will therefore produce oscillations; the observed frequency of a mode places only a single constraint on a combination of $v_{\phi}$ and $v_{\theta}$ on the integer $q$ surfaces, but the ease and accuracy of the measurement make it a valuable adjunct and check for inferences of the bulk fluid velocity. The rotation velocity also affects the phase velocity of the various small-scale instabilities in the plasma. These will be discussed in Sec. VI.A; the effect is a supplementary source of rotation information.

\section{E. Current density}

The final fluid quantity required is the current density, which enters the Grad-Shafranov equation for determining the equilibrium configuration. The current is largely in the toroidal direction, and $j, j_{\phi}$, and $j_{\|}$are used interchangeably. Since the current is carried by electrons, one would think first of measuring the electron drift velocity, as, for example, in the Doppler shift of the Thomson-scattering spectrum. However, the drift velocities are typically small $\left(v_{d}<0.1 v_{\text {th }}\right)$. The minuteness of the Thomson-scattering cross section and geometries which observe only $v_{\perp}$ preclude useful determinations. (Measurements at this level are routinely obtained spectroscopically for rotation velocity, where the line intensities are higher.) Instead, all inferences of $j$ are based on determinations of $B_{\theta}$ :

$$
B_{\theta}(r)=\mu_{0} \frac{1}{r} \int_{0}^{r} j\left(r^{\prime}\right) r^{\prime} d r^{\prime}
$$

for the simple cylindrical approximation, and a more complicated integral, which must generally be done numerically, for more complex configurations. The awkward property of Eq. (4.5) is that the inversion to find $j$ requires differentiation and therefore high accuracy in the $B_{\theta}$ measurement. Fortunately, the value of $B_{\theta}$ itself is significant; for example, $q$ is directly proportional to $B_{\theta}$.

The first technique to be systematically developed is closely related to interferometry. A circularly polarized electromagnetic wave suffers a polarization rotation, subject to the same requirements as those that apply to density interferometry, proportional to $\int n B_{\|} d l$, where $B_{\|}$here means the component of $B$ along the propagation direction $d l$. As with density measurements, great care is required to invert a set of

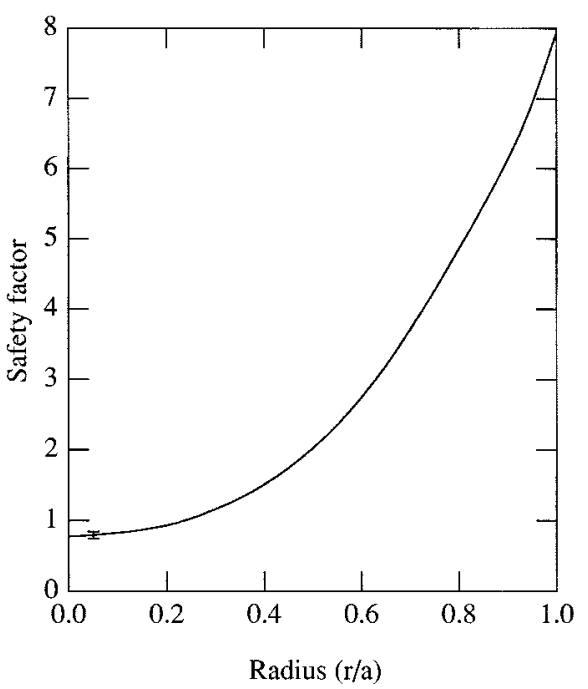

FIG. 19. Safety factor $q(\rho)$ profile from TFTR using the motional Stark effect. Only one representative error bar is shown. The measured $q$ profiles support inferences based on neoclassical resistivity and measured electron temperature (Levinton et al., 1993).

line integrals to infer $B_{\theta}$. The technique was first applied on TEXTOR (at Julich, Germany); results are described by Soltwisch (1988). Although the technique is technically difficult, it requires only modifications to a multichannel interferometer system, which is provided on most tokamaks. A recent improvement in technique is illustrated by Rice and Hooper (1994).

An obvious approach would be to use the Zeeman effect in some arrangement to measure the magnetic-field direction, but exploiting this effect has proved quite difficult. West et al. (1987), using lithium beams, and Marmar et al. (1989), using lithium pellets, among others, have obtained $q$ profiles, but the details are too complex to be included here. Profiles have also been obtained using the motional Stark effect (measuring the electric field produced by $B_{\theta}$ in the frame of a moving atom-typically the atoms in a heating beam). For hydrogen energies of $\sim 100 \mathrm{keV}$, the Stark splitting of a few angstroms is comparatively easy to measure. The technique is described by Levinton (1992), with typical results from TFTR shown in Fig. 19 (Levinton et al., 1993). As a direct measurement of $B_{\theta}$, it gives relatively direct and accurate results for $q(\rho)$, which are illustrated in Fig. 19. The widespread use of heating beams makes the technique broadly applicable.

Intensive development of all techniques to measure current profiles is continuing, and several other approaches are being explored but have not yet reached regular use. All the techniques require considerable care in application and interpretation. The current or $B_{\theta}$ profile remains the fluid parameter measured with the least regularity and resolution.

Given the difficulty of obtaining full profiles of $j$ or $B_{\theta}$ directly, several indirect methods are important. Traditionally, the current profile has been inferred from the $T_{e}$ profile, assuming that the resistivity was classical (Spitzer) or neoclassical. Unlike transport across flux surfaces, which is rarely neoclassical, parallel transport appears to follow neoclassical theory. The bottom row of Eq. (2.4) has been reasonably confirmed for both diagonal and off-diagonal ele- 
ments (cf. Bartlett et al., 1988 and Zarnstorff et al., 1990). The only additional experimental parameter that is required for the neoclassical calculation of $j$ is $Z_{\text {eff }}$, an average effective ion charge. The resistivity depends directly on the electron collision rate with ions. Because the cross section varies with $Z^{2}$, even a small concentration of high- $Z$ ions can increase the collision rate. The definition of $Z_{\text {eff }}$ is

$$
Z_{\text {eff }}=\frac{\sum n_{i} Z_{i}^{2}}{\sum n_{i} Z_{i}}, \quad n=\sum n_{i} Z_{i},
$$

where the sum extends over all ionization states of all ion species present, and $n$ is the usual fluid (electron) density. Although one might imagine determining all the $n_{i}$ from absolute spectroscopic measurements, the diversity of elements and charge states is usually excessive. (The exception is a plasma dominated by a single, fully stripped impurity. An example is carbon in TFTR as described by Synakowski et al., 1993.) Instead, a useful first approximation assumes $Z_{\text {eff }}$ uniform across the plasma and adjusts the value for agreement of the temperature profile and plasma current with the loop voltage. Of course, this does nothing to establish the validity of neoclassical conductivity. To test neoclassical conductivity and provide more accurate analyses, an independent measure of $Z_{\text {eff }}(r)$ is required. Perhaps the best diagnostic for this purpose measures the bremsstrahlung continuum emission in the visible arising from electron collisions with the various ions. Care is required in order to avoid emission lines, and the intensity is far below blackbody; but the local emissivity is simply proportional to $n^{2} Z_{\text {eff }} \sqrt{T_{e}}$. Measurements of absolute intensity for a number of chords through the plasma coupled with $n$ and $T_{e}$ profiles can be inverted to determine $Z_{\text {eff }}(r)$. Although such inversions are sensitive to error, the observations generally find a self-consistent, relatively flat $Z_{\text {eff }}(r)$, and the value is consistent with neoclassical calculations.

Additional information and constraints are obtained from observation of the MHD activity mentioned in the previous section. These modes originate on rational $q$ surfaces, $q$ $=m / n$. If the poloidal and toroidal mode numbers $(m, n)$ can be ascertained and the radius found at which the mode occurs, one point of $q(r)$ can thereby be determined. These points may be compared with direct $q$ profiles or used as constraints in the calculation of $j(r)$ from $T_{e}(r)$. The most important of these MHD modes is the sawtooth oscillation, which appears as a strong modulation on soft $\mathrm{x}$-ray and ECE signals. Both subsidiary $m=1, n=1$ activity and phase reversal of the large sawtooth signal are presumed to mark the $q=1$ surface. Diagnostic techniques for sawteeth and related phenomena have been separately reviewed by Costley (1988). The diagnostic objectives are not merely to constrain the $q(r)$ profile, but to elucidate the physics of the oscillations.

\section{TRANSPORT}

The objective of transport analysis is to determine the transport coefficients as typified by the matrix of Eq. (2.4). Conservation equations for particles, electron energy, ion energy, etc. have been written, including transport, and sufficient experimental data are sought to permit inference of the transport coefficients. These analyses are extremely complex; here we are concerned primarily with the diagnostics necessary to provide the input data. These data certainly include the fluid parameters of Sec. IV. Moreover, the appearance of the derivatives $d n / d r$ and $d T / d r$ in Eq. (2.4) implies that the density and temperature profiles must be obtained with sufficient resolution and accuracy to define these quantities. Some additional quantities also enter the equations. Since the primary criterion for a fusion reactor is sufficient energy confinement, we consider energy transport first.

\section{A. Energy}

A representative energy conservation equation for electrons in equilibrium may be written as

$$
\begin{gathered}
\frac{3}{2} \frac{\partial\left(n T_{e}\right)}{\partial t}-\frac{1}{r} \frac{\partial}{\partial r}\left(r \kappa \frac{\partial T_{e}}{\partial r}\right)+\frac{1}{r} \frac{d}{d r}\left(r \frac{5}{2} k T_{e} \Gamma\right) \\
=\frac{E^{2}}{\eta}-n \nu_{e i}\left(T_{e}-T_{i}\right)+P_{\mathrm{in}}-P_{\mathrm{rad}},
\end{gathered}
$$

where the terms on the left are the time derivative of energy content and the divergence of the energy flux from a simplified transport matrix including only the diagonal thermal conductivity and particle convection. The right-hand side includes the energy inputs from Ohmic heating, with $\eta$ being Spitzer or neoclassical resistivity, and other sources $P_{\text {in }}$ (e.g., beams or rf) and energy losses to ions by collisions and to radiation (largely inelastic collisions with impurities). The analysis is generally applied to an equilibrium state, for which the time dependence vanishes and time averaging may be used to improve accuracy. To avoid second derivatives, Eq. (5.1) is usually employed in the form

$$
\begin{aligned}
-\kappa_{e} \frac{d T_{e}}{d r}= & -\frac{5}{2} k T_{e} \Gamma+\frac{1}{r} \int_{0}^{r} r d r\left[\frac{E^{2}}{\eta}-n \nu_{e i}\left(T_{e}-T_{i}\right)\right. \\
& \left.-P_{\text {rad }}+P_{\text {in }}\right]
\end{aligned}
$$

from which $\kappa$ may be extracted if all other quantities are measured. For convenience in comparing various diagonal terms, a $\chi_{e}\left(\kappa_{e}=n \chi_{e}\right)$ with units of $\mathrm{m}^{2} / s$ is often used as the transport coefficient. Taking the terms in order, we have discussed the temperature profile, and the particle flux $\Gamma$ will be examined in Sec. V.B. However, it is often a small term in the interior of the plasma, for particle sources are often absent or small. The electric field follows immediately from the loop voltage, and the resistivity is neoclassical, as discussed above. The electron-ion exchange is more subtle, because if $\nu_{e i}$ is significant, a frequent case, $T_{e}$ and $T_{i}$ must each be determined with considerable precision. From the earlier discussion, it is clear that they are measured with quite different techniques; both random and systematic errors will enter the comparison. This term often contributes the major uncertainty in $\chi_{e}$. A partial solution to this problem is discussed below. The radiation loss $P_{\text {rad }}$ is measured independently. Again, one can obtain estimates from spectroscopic measurements of impurities, because the important contribution is entirely line radiation; but the most useful data come from bolometry, direct measurements of total radiated power along 
multiple sight lines through the plasma that are inverted to give $P_{\text {rad }}(r)$. Until recently, these measurements depended on thin foil thermistors (Muller et al., 1982; Mast and Krause, 1985; Schivell, 1985). Bolometry has the advantage of simple calibration and uniform sensitivity over the full wavelength range over which significant radiation is emitted. A lack of sensitivity precludes fast time resolution, although this does not affect their use in equilibrium measurements, as in Eq. (5.2). Recently, solid-state diode detectors have been employed for these measurements. They offer high sensitivity and fast time response, but their response is limited to wavelengths shorter than visible and is somewhat nonuniform. Nevertheless, their range of sensitivity is often well suited to the actual plasma emission spectrum, and the technique is quite valuable, especially in extending $P_{\text {rad }}$ measurements to fluctuations (Wen and Bravenec, 1995).

Evaluation of $P_{\text {in }}(r)$ is probably the most complex task. In modern tokamaks with strong auxiliary heating, it is as large as any other term in Eq. (5.2), and a direct experimental measurement is not generally possible. Instead, elaborate calculation based on the specific physics of the heating process must be employed. To illustrate, we shall consider three important heating techniques: neutral beams, rf heating near the ion cyclotron frequency, and if heating at the electron cyclotron frequency.

Neutral beams were the first strong auxiliary heating source in tokamaks. The beam is produced by extracting an ion current $(\sim 10 \mathrm{~A})$ from a plasma source, accelerating it (50 to $120 \mathrm{kV}$ ), passing it through gas sufficient to reach approximate $50 \%$ equilibrium between ions and neutrals, deflecting the ions magnetically, and directing the neutral beam through the plasma. Neutral beams represent a major technological development; single beams can deliver several megawatts of neutral-beam power. The incident power can be measured directly by calorimetry; and power not absorbed ("shine-through"), likewise, albeit with lesser accuracy. However, $P_{\text {in }}(r)$ is complicated because some of the neutrals ionize on orbits which leave the plasma promptly; all the orbits have to be computed carefully; and the energetic ions transfer their energy differently to ions and electrons as they slow. [The $P_{\text {in }}(r)$ term in Eq. (5.2) is only the direct transfer to electrons.] Many elements of the slowing down of energetic ions due to classical collisions have been carefully confirmed experimentally (by examining the evolution of the ion distribution function as discussed in Sec. IV.C), but a major calculation specific to the beam configuration and plasma parameters must be employed to obtain $P_{\text {in }}(r)$ for determining $\chi_{e}$.

The use of rf heating, for example, at frequencies related to the ion cyclotron frequency, is equally complex. The vacuum wavelengths exceed the plasma size, the "propagation" within the plasma is complicated, and the absorption processes result from nontrivial kinetic effects. Although the power coupled out of the antenna at the plasma boundary can be determined by standard engineering measurements, the determination of $P_{\text {in }}(r)$ is at least as difficult as that for beam heating. Since the absorption often results in the production of highly energetic ions, there can be an important component of direct electron heating.
Heating at the electron cyclotron frequency is the simplest, at least from the perspective of analysis. Calculation of wave propagation through the plasma is comparatively straightforward; geometrical optics is a good approximation. The absorption is entirely on electrons, and the calculation is comparatively easy. Furthermore, a measure of direct confirmation may be obtained by examining local heating rates when the power is first applied. The initial rates of temperature rise indicate absorbed power for times short compared with transport time scales.

The equation for ion thermal conductivity or $\chi_{i}$ $\left(\kappa_{i}=n \chi_{i}\right)$ corresponding to Eq. (5.2) may be written

$$
-\kappa_{i} \frac{d T_{i}}{d r}=-\frac{5}{2} k T_{i} \Gamma+\frac{1}{r} \int_{0}^{r} r d r\left[n \nu_{e i}\left(T_{e}-T_{i}\right)+P_{\mathrm{in}}\right]
$$

where the $P_{\text {in }}$ has the analogous meaning but differs numerically from Eq. (5.2). Here, $P_{\text {in }}$ includes direct ion heating from beams and rf heating less charge-exchange losses. Application poses the same problems discussed above for the determination of $\kappa_{e}, \chi_{e}$. The convection term $\Gamma$ is more often important in Eq. (5.3), and some care is required in plasmas with significant impurity content, for which $T_{i}$ may not equal the $T_{\text {imp }}$ that are often measured to infer $T_{i}$. Additional equations may be needed. However, if the data have been obtained to determine one $\chi$, the other can usually be determined also.

A frequent problem concerns the ion-electron energy transfer mentioned above. If $T_{e} \sim T_{i}$, but the transfer term is too large for independent calculation of $\chi_{e}$ and $\chi_{i}$, Eqs. (5.2) and (5.3) may be added to include the total-energy content:

$$
-\kappa \frac{d T}{d r}=-5 k T \Gamma+\frac{1}{r} \int_{0}^{r} r d r\left[\frac{E^{2}}{\eta}-P_{\mathrm{rad}}+P_{\mathrm{in}}\right],
$$

from which one obtains a single $\kappa$ or $\chi$. This requires fewer temperature data, since a good $T_{e}(r)$ suffices, and even the $P_{\text {in }}(r)$ is somewhat easier to calculate because separation of ion and electron contributions is unnecessary. The penalty is loss of physical insight. Insofar as $\chi_{e}$ and $\chi_{i}$ have been independently determined, they have not been found to be simply related.

The application of Eqs. (5.1)-(5.3) for the determination of thermal conductivity requires further discussion. One cannot simply evaluate the derivative on the left by differencing adjacent points on the temperature profile and attempt to evaluate the terms on the right at the average position. The disparate accuracy, resolution, and grid spacing of the various elements make that impractical and inaccurate. Satisfactory evaluation of transport coefficients has depended not only on the development of extensive profile diagnostics, but also on the development of complex analysis codes. Although these are not properly diagnostics, their importance to extracting physically significant information from experimental data justifies a brief discussion in this context. The basic codes integrate data from all diagnostics to infer reasonable, smooth profiles for all quantities in Eqs. (5.2) and (5.3), from which a smooth profile of $\kappa$ or $\chi$ may be deduced. Implicit in the analysis is the assumption that there is no small-scale or strong local variation in transport. (In Fourier analysis, it would correspond to keeping only the first 


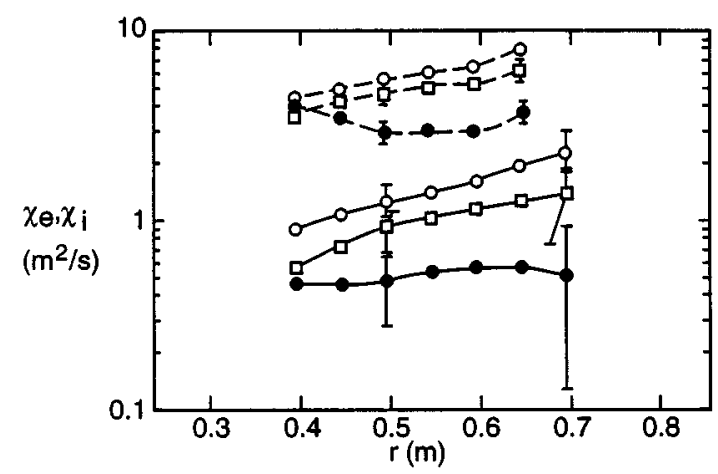

FIG. 20. Thermal diffusivity profiles from TFTR. Both $\chi_{i}$ (dashed line) and $\chi_{e}$ (solid line) are shown, increasing with heating power of 4.5, 9, and $14 \mathrm{MW}$ (Efthimion et al., 1991).

few terms; in these analyses, it is more often applied by using spline fits with only a limited number of independent points on the profile.) Typical of these codes is SNAP (Towner et al., 1992). An important practical problem is that some discharges, especially those with exceptional properties, never reach a time-independent steady state as assumed in Eqs. (5.2)-(5.4). The equations can easily be corrected by adding the appropriate time derivatives, as in Eq. (5.1), to the integrals on the right-hand side, but more elaborate timedependent calculations are also required for $E(r, t)$ and $P_{\text {in }}$. The most widely used of the full time-dependent transport codes is TRANSP, developed at Princeton (Goldston et al., 1981), which enables one to obtain transport coefficients as the plasma is changed in various ways with almost any form of applied heating. ONE-TWO is a similar code run on DIII-D (Pfeiffer et al., 1985).

Representative results for the radial profiles of thermal diffusivity are shown in Figs. 20 and 21. Figure 20 is from an experiment on TFTR to measure the increase in transport coefficients with increasing auxiliary heating power and temperature. The increase in thermal diffusivity is naturally associated with a decrease in energy confinement time $\left[\tau_{E}\right.$ of Eq. (2.9)]. Although decreases in $\tau_{E}$ with increasing heating have been found in all tokamaks, some operating regimes have been found that minimize the effect. Perhaps the most important of these is the H-mode, of which the steep density gradient at the edge (Fig. 10) is a unique characteristic. Figure 21 gives the energy transport coefficients for a typical H-mode condition from DIII-D. Regimes of improved confinement often include regions of steep gradients that challenge diagnostic capability.

The strong radial variations of the transport coefficients in Figs. 20 and 21 are typical, as are the error bars or bands. Since the evaluations of transport coefficients are not point calculations but reflect averages and fits over a plasma region, error bands are appropriate and informative, although they lack the precise definitions of 1 or $2 \sigma$ bounds on the measurement of a random variable. The important conclusion is that energy transport can be characterized by local transport coefficients determined with reasonable accuracy. These results exclude both the core and the edge of the plasma. Equations (5.2) and (5.3) are difficult to apply in the core because the gradients approach zero at the center, and time variations such as sawteeth confuse the interpretation. Near the edge, strong radiative and charge-exchange losses,

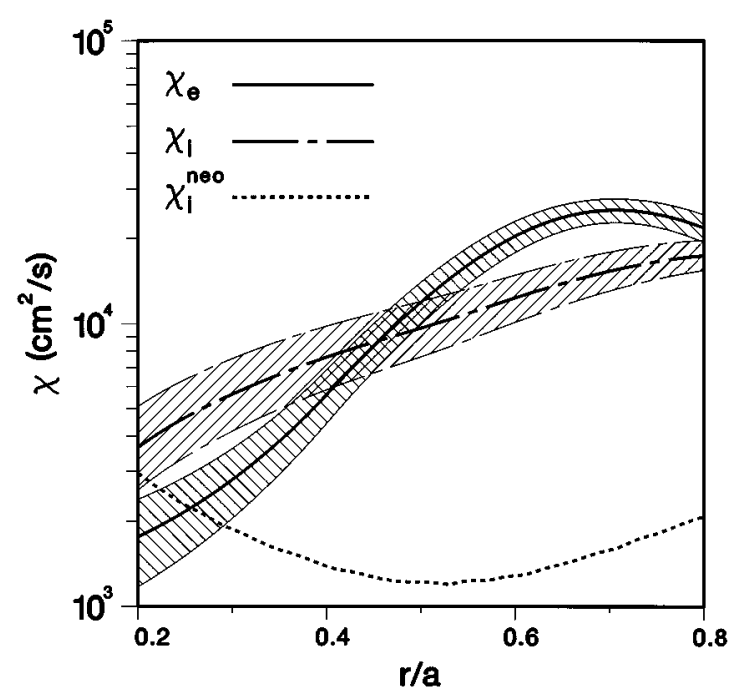

FIG. 21. Thermal diffusivity profiles from DIII-D. The smooth curves with error bands are a good representation of the results and uncertainties in transport analyses. The inadequacy of neoclassical transport theory is illustrated by the ion prediction shown. This is an $\mathrm{H}$-mode discharge at a plasma current of $1.35 \mathrm{MA}$ with $6 \mathrm{MW}$ of neutral-beam heating (Petty, 1995).

as well as a growing convective term $\Gamma$, greatly decrease accuracy.

\section{B. Particles}

The particle conservation equation is simpler than the energy transport equation in many respects, but it includes an essential complication. The equilibrium density equation is

$$
D(r) \frac{\partial n}{\partial r}+V(r) n=-\frac{1}{r} \int_{0}^{r} S\left(r^{\prime}\right) r^{\prime} d r^{\prime},
$$

where $S$ is the source from ionization, and $V$ represents all the off-diagonal terms in Eq. (2.4). Such off-diagonal terms were omitted in the energy equations for simplicity, an application of Occam's razor. Except for some recent experiments with ECH heating, it has always been possible to analyze equilibrium transport with only a $\chi$, because the lefthand sides of Eqs. (5.2) and (5.3) were always positive. However, neutral atoms originating outside the plasma can penetrate only a short distance into hot, dense fusion plasmas. In the absence of neutral-beam heating, $S(r) \sim 0$ except at the edge. Lacking a $V$, the implication would be that $d n / d r \sim 0$, a flat density profile. Density profiles are often reasonably peaked and rarely as flat as $V=0$ would require. Neoclassical theory does predict an inward $V$, but usually not sufficient to explain experimental profiles. In the absence of sources, one obtains

$$
\frac{1}{n} \frac{d n}{d r}=-\frac{V}{D},
$$

a good experimental measure of $V / D$, but no measure of magnitudes. From measurements of the source, specifically evaluated out to the edge where the density is low and the density gradient is large, one can obtain 


$$
D(a) \frac{\partial n}{\partial r} \approx-\frac{1}{a} \int_{0}^{a} S\left(r^{\prime}\right) r^{\prime} d r^{\prime}
$$

as an estimate of $D$ at the edge. However, the measurement of $S(r)$ is quite difficult. There are good data for the relation between ionization rate and the intensity, for example, of $H_{\alpha}$ emission for the relevant ranges of plasma density and temperature. Although absolute measurements of $H_{\alpha}$ intensity are reasonable spectroscopic practice, the emission lacks the poloidal and toroidal symmetry that reduces the problem of measuring most other plasma parameters to a single $f(r)$. Extensive measurements and some modeling are required to obtain the (flux-surface average) $S(r)$ that enters Eqs. (5.5) and (5.7).

As a consequence, equilibrium particle transport is less often analyzed and particle transport coefficients are less widely known than are energy transport coefficients. The separation and determination of diagonal and off-diagonal terms can only be made in time-dependent experiments (cf. Sec. V.D).

Although two-fluid equations are a minimal description, the strong requirement of charge neutrality (cf. $\lambda_{D}$ in Table I) implies $n_{e}=\sum n_{i} Z_{i}$. Unless the impurity concentration is large, a single transport equation suffices for both electrons and the principal ion species. The transport of impurities is an independent, important question. Besides its interest as a sort of test-particle diffusion, impurity transport is practically important for determining how easily helium "ash" from fusion reactions will leave the plasma and how strongly heavy impurities, which will dilute the hydrogen and possibly radiate, will accumulate in the plasma. However, there are two major problems in obtaining impurity transport coefficients. First, as discussed in Sec. IV.E, measurement of impurity concentration is difficult. Second, just as with Eq. (5.5), the equilibrium impurity profile fixes only $V / D$ [Eq. (5.6)]. The separate transport coefficients can be extracted only from time-dependent experiments.

Spectroscopic measurement of impurities is far more important than merely as a contributor to $Z_{\text {eff }}$ and radiated power measurements and impurity transport studies. In emphasizing tokamak physics, we have neglected the art and engineering of modern tokamaks. As a practical matter, good performance requires low levels of impurities and radiated power. Spectroscopic impurity monitoring has been essential to developing successful configurations, materials, and operating regimes as well as to detecting malfunctions.

\section{Momentum}

Since most particles can stream freely along magneticfield lines in a tokamak, the momentum density $p_{\|}\left(\sim p_{\phi}\right)$ is an independent thermodynamic variable. Energetic neutral beams, if not directed exactly perpendicularly to the magnetic field, can easily impart a substantial toroidal momentum as well as energy to the plasma. Given a strong source, measurement of the plasma drift velocity can then be used in equations analogous to Eq. (5.3) to obtain a $\chi_{p}$. The value is of particular interest for comparison with $\chi_{i}$, because processes that transport one would generally be expected to

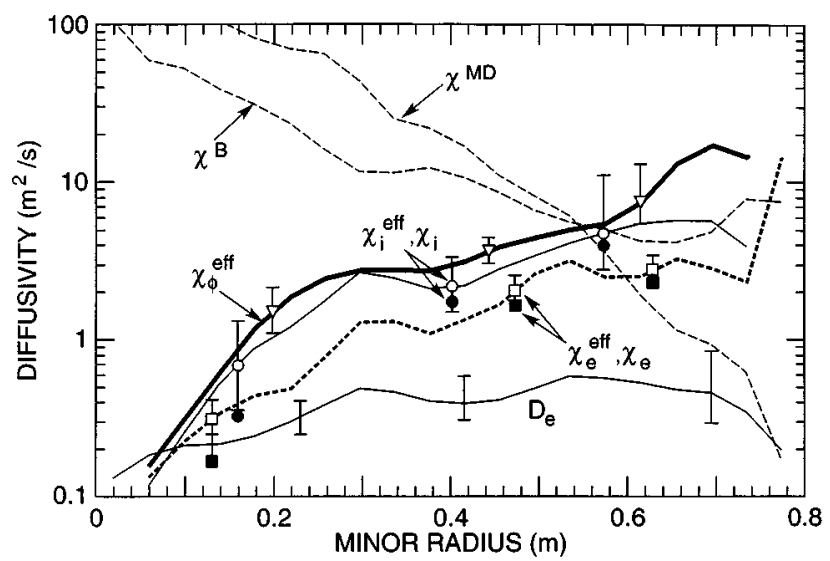

FIG. 22. Transport coefficients in TFTR. The minor radius is $0.8 \mathrm{~m}$; the horizontal axis corresponds closely to a linear $0 \leqslant \rho \leqslant 1.0$. [Figure $4(\mathrm{a})$ is a good picture of the magnetic configuration for this case.] In addition to the momentum diffusivity $\chi_{\phi}$, the ion and electron diffusivities, $\chi_{i}$ and $\chi_{e}$, are shown, together with an estimate for the particle $D$ and two theoretical models, labeled $\chi^{B}$ and $\chi^{M D}$. This analysis is continued closer to both the center and edge than is often possible. The small gradients near the center and high radiation near the edge increase errors in those regions. The tendencies for diffusivities to rise toward the edge is a general phenomenon (Scott et al., 1990).

transport the other, and the $\chi_{p}$ measurement is independent of uncertainties in the $n \nu_{e i}\left(T_{e}-T_{i}\right)$ term of Eq. (5.3).

A good example of comprehensive transport analysis is shown in Fig. 22 (Scott et al., 1990) for a strongly beamheated discharge in TFTR. [The notation $\chi^{\text {eff }}$ indicates that the $\Gamma$ terms in Eqs. (5.2) and (5.3) have been omitted from the calculation. The estimate for $D$ was obtained by using the beam particle source in Eq. (5.7), neglecting $V$.] One can obtain a good picture of the transport processes with reasonable resolution and accuracy.

\section{Time-dependent transport}

Time dependence has been mentioned in the context of requiring corrections to simple steady-state transport calculations, but its significance is far broader. From a fundamental viewpoint, characterizing the response of a physical system to perturbations is as significant and informative as describing the equilibrium state. For systems in which transport is caused by collisions and for which the resulting transport coefficients typically depend weakly on local parameters, transient and equilibrium analyses are complementary methods for measuring the coefficients. However, the transport rates in hot plasmas are far above collisional (neoclassical) values; they are presumably driven by turbulent processes that depend strongly on plasma parameters, a presumption supported by the strong radial variation in transport coefficients as shown, for example, in Fig. 22. One consequence of transport coefficients with strong parametric dependencies is that if the equilibrium transport equation is linearized for perturbations, the transport coefficients that appear in the equation for a linear perturbation will differ from those evaluated at equilibrium (see, for example, the explicit expressions in Gentle, 1988). A second consequence is that 


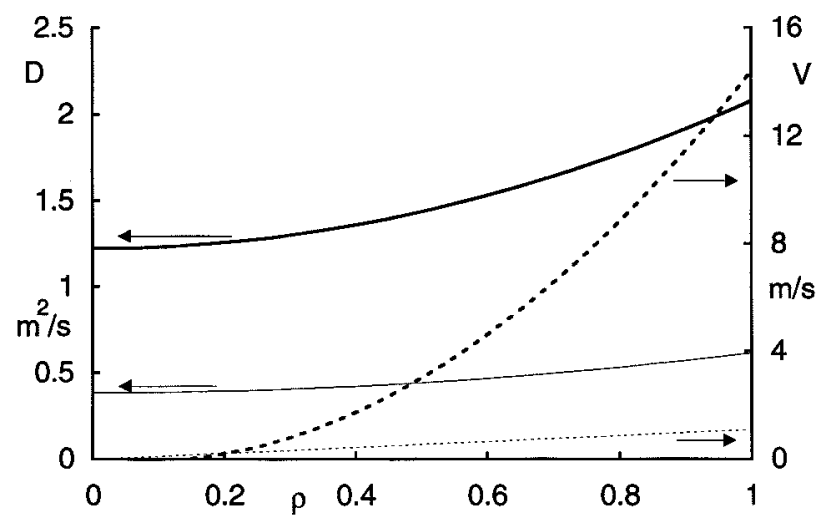

FIG. 23. Particle transport coefficients from ASDEX Ohmic discharges using perturbations. The heavy lines are from a low-density case $\left(1.0 \times 10^{19} \mathrm{~m}^{-3}\right)$, and the light lines are at higher density $\left(4.0 \times 10^{19} \mathrm{~m}^{-3}\right)$. The diffusion coefficients are solid lines; the inward convective velocity, dashed. Error bands (not shown) are $\pm 30 \%$.

the equations for density and temperature perturbations, and possibly other quantities, will generally be coupled.

A review of analysis techniques and results for transient transport is far beyond the scope of this review. Suffice it to say that it is a very complex and fruitful area, especially for particle transport, where equilibrium analysis gives limited information, as discussed above, and for exploring coupling and off-diagonal terms. This area is included in the review by Burrell et al. (1990). From the diagnostic viewpoint, these experiments require good space and time resolution for measurement of perturbed quantities. The quality and quantity of this work has expanded dramatically over the past decade because of diagnostic improvements.

For density, multichord interferometers are the principal tool. They easily provide a continuous $\int n(t) d l$ with sufficient time resolution; the important improvements have been in number of channels and especially in precision to detect perturbations as small as $\sim 5 \%$ with good accuracy. An example of particle transport coefficients measured in ASDEX (at Max-Planck-Institute, Garching, Germany) (Gentle et al., 1992) by density modulation is shown in Fig. 23. Similar results from TFTR using gas puffs are shown in Fig. 24 (Efthimion et al., 1991). The plots show $D$ and $V$, but the labels are chosen to emphasize that the coefficients apply to the perturbation, not to the equilibrium.

Reflectometry has proven especially valuable for perturbation studies. It gives a comparatively direct indication of local $\Delta n(t)$, obviating inversions of chordal data. Difficulties in constructing complete equilibrium $n(r)$ profiles are irrelevant for the transient measurements.

Impurity transients for transport analysis may be produced by gas injection of volatile materials or by laser ablation from a target near the plasma. Considerable information can be adduced using only the time histories of a few ionization states (chosen to occupy different radial positions in the plasma) without measuring absolute concentrations of all states. The most complete analyses have used helium as a trace impurity in a hydrogen plasma, for which only $\mathrm{He}^{++}$ need be followed using charge-exchange spectroscopy. This technique can also be applied to carbon and oxygen transport because they are fully stripped over most of the cross section

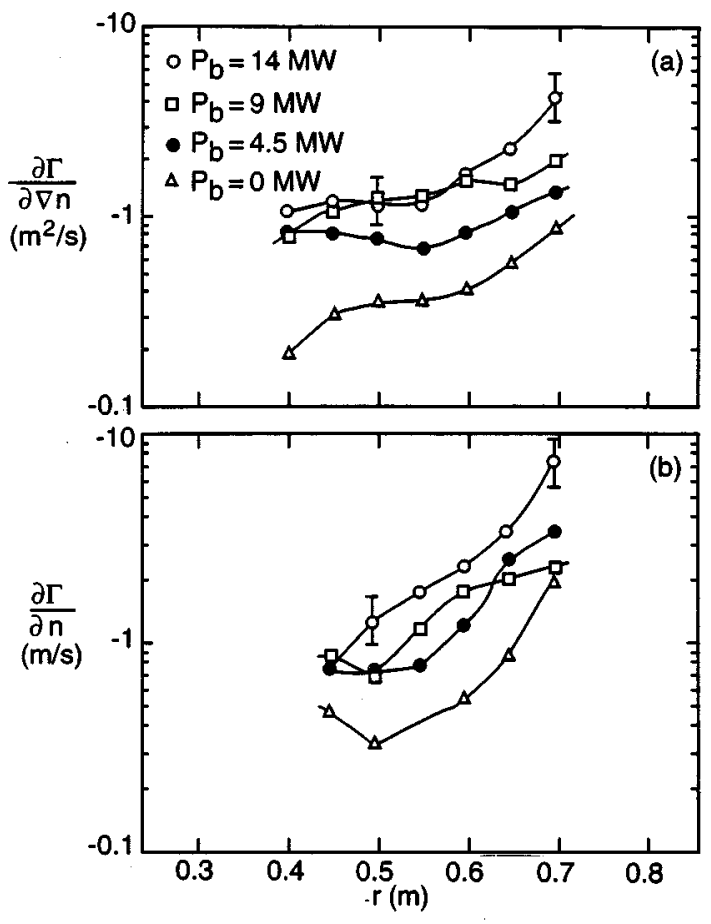

FIG. 24. TFTR particle transport coefficients from density perturbations (Efthimion et al., 1991): (a) diffusion coefficient for the perturbation; (b) convective velocity for the perturbation, negative inward.

in large, hot tokamaks. However, impurity transport experiments are optimally performed using an element not normally present. Synakowski et al. (1993) provide a recent example including several of these techniques, shown in Fig. 25. (The $D_{e}^{\text {eff }}$ is the same estimate of $D_{e}$ discussed with Fig. 22 ; inward convection is neglected.)

For temperature, Thomson scattering cannot provide a continuous time history, but ECE systems, specifically heterodyne or grating systems, provide excellent data. Historically, measurements of soft-x-ray emission obtained with diodes sensitive roughly from $500 \mathrm{eV}$ to $5 \mathrm{keV}$ have also been very important. Dozens of chords with time resolution approaching $1 \mu \mathrm{sec}$ are easily provided. The value of the method is limited only by the difficulty of interpretation. The radiation in this range is a combination of bremsstrahlung continuum and line radiation from high- $Z$ impurities $(\mathrm{Fe}, \mathrm{Ni}$, etc.) and depends upon electron density, impurity content, and strongly on $T_{e}$. Although it is a chordal measurement, its strong $T$ dependence implies that the signal is dominated by the highest temperature along the sight line. However, the sensitive dependence on impurities makes it almost impossible to extract $T_{e}$. Instead, the signals may be used as an indication of $\Delta T(t)$ to study the propagation of transients. A second use is possible because the diodes can be easily set into arrays with multiple sight lines through the plasma as required for tomography. Although the $\mathrm{x}$-ray emissivity is a complex function of local variables, they are each flux functions; the emissivity is constant on flux surfaces. Hence the data may be inverted tomographically to produce an image of interior flux surfaces both as confirmation of the magnetic calculation and as an indication of special structures, instabilities, and perturbations (Granetz and Smeulders, 1988). Examples of these techniques for determining complex inter- 

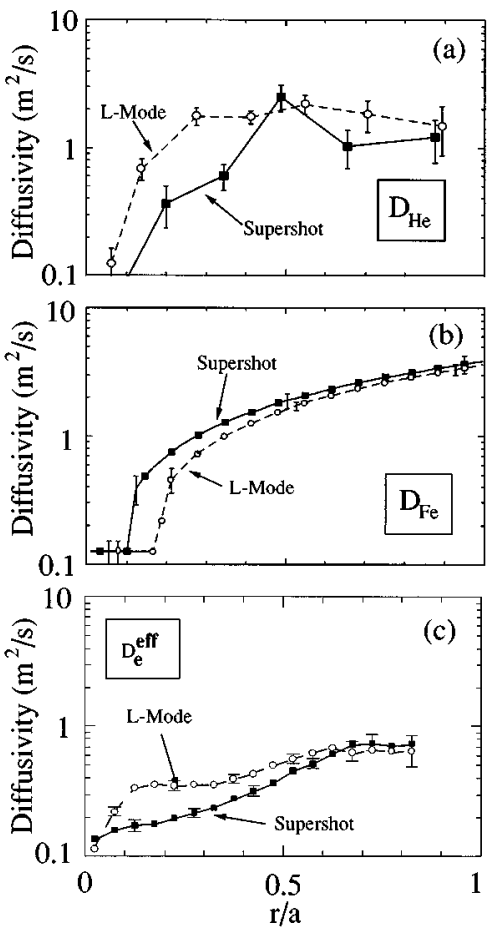

FIG. 25. Impurity diffusion coefficients for helium and iron on TFTR, including an estimate of the bulk particle diffusion $D_{e}$ with the same meaning as in Fig. 22 (Synakowski et al., 1993).

nal magnetic structures on JET are shown in Figs. 26 and 27 (Costley, 1988). Figure 26 shows results from an ECE reconstruction of isothermal surfaces, whereas Fig. 27 shows surfaces of constant $\mathrm{x}$-ray emissivity at the same time. Both techniques provide good images of the flux contours, in this case a more complex topology than that of Fig. 1. The region shown in Figs. 26 and 27 includes only $\rho<0.5$.

Both x-ray emission and ECE are also used to study timedependent transport. Historically, the first studies of this type analyzed the x-ray emission from sawteeth, relaxation oscillations that often occur naturally in the central region of tokamaks and produce an oscillating temperature that diffuses outward (Jahns et al., 1978). Now, multichannel ECE systems provide absolute $T_{e}(r, t)$ data for all sorts of perturbations which are susceptible to much more precise analysis. The work of Gorini et al. (1993), which examines both natural sawtooth oscillations and temperature perturbations driven by modulated heating, is typical, and the field is expanding rapidly (Kissick et al., 1994; Gentle et al., 1995).

Unfortunately, the techniques for measuring ion temperature require such long integration times to obtain good signals that they cannot provide data for fast time dependencies or fluctuations.

\section{FLUCTUATIONS}

Fluctuations-deviations in space and time of the local density, temperature, etc. from the flux-surface averaged fluid value-are important because of their role in the ubiquitous "anomalous transport" in hot plasmas, transport at rates far above the neoclassical collisional rates. The enhanced rates, observed for the transport across flux surfaces of all fluid quantities, are presumably caused by some sort of

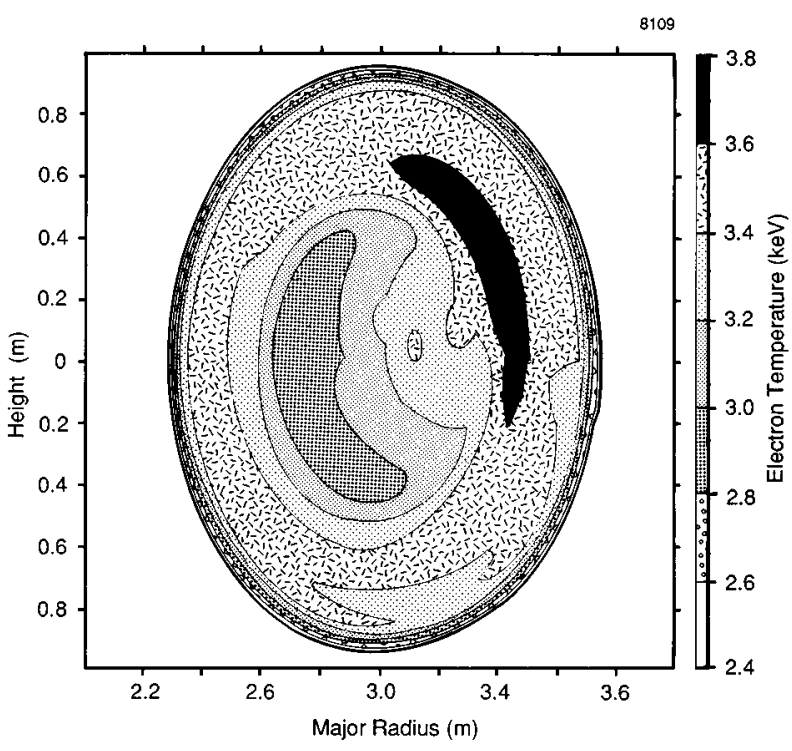

G88.178/1

FIG. 26. Temperature contours derived from ECE $2 \mathrm{~ms}$ after a sawtooth crash in JET. Only the central region is shown. The temperature is no longer poloidally symmetric or peaked at the center, but shows a complex structure (Costley, 1988).

turbulent fluctuations. Theoretically there is no dearth of candidate instabilities, but none has proved equal to the task of predicting transport observations. There are two sorts of diagnostic objectives. The simplest, most immediate goal is to observe the fluctuations and provide some characterizationamplitude, frequency spectrum, wavelength range, etc. The second, far more subtle and difficult, is to obtain all information needed to evaluate the fluctuation-driven transport as in Eqs. (2.6) and (2.7). These require the simultaneous observation of at least two fluctuating quantities at a single point,

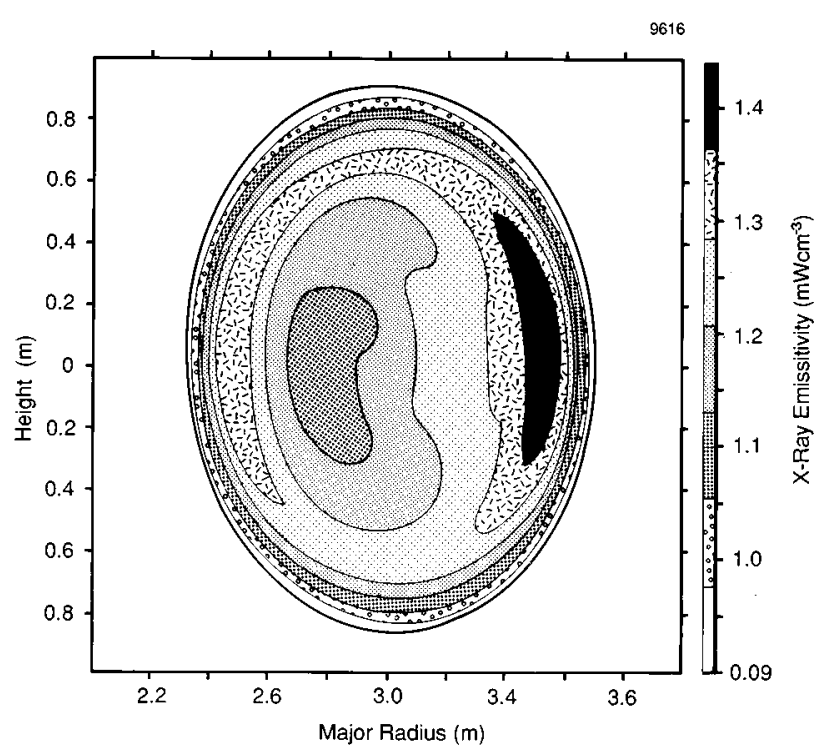

G88.178/2

FIG. 27. Contours of x-ray emissivity from JET for conditions similar to those of Fig. 26 (Costley, 1988). 
including their correlation properties. Moreover, the radial flux is not a flux-surface quantity; it can be expected to vary poloidally, if not toroidally. Considerable progress has been made toward the first objective, but the second remains incomplete, except in the plasma edge region under conditions accessible by material probes.

\section{A. Density}

There are several complementary techniques for measuring plasma density fluctuations, but no single complete method. The most elegant is that based on collective scattering of electromagnetic radiation. Under the same conditions as those that apply to density interferometry, a fluctuating density $\tilde{n}\left(\omega_{w}, \mathbf{k}_{w}\right)$ in Fourier representation produces a corresponding fluctuation in the index of refraction of the medium. An incoming wave $\omega_{i}, \mathbf{k}_{i}$ is scattered to $\omega_{s}, \mathbf{k}_{s}$ with $\Delta \omega=\omega_{w}$ and $\Delta \mathbf{k}=\mathbf{k}_{w}$. The optics of the input and output beams determine the observation region and $\Delta \mathbf{k}$; a heterodyne detector gives the frequency spectrum. The system can be calibrated to provide $\tilde{n}\left(\omega_{w}, \mathbf{k}_{w}\right)$. The schematic of a typical system is shown in Fig. 28, the configuration on TEXT (Brower et al., 1987).

Representative results from this system are given in Fig. 29 (Brower et al., 1988), indicating the range of $k_{\theta}$ measured and the heterodyne method to distinguish direction of propagation by the sign of the frequency shift. Although the physics of tokamak plasmas is well beyond the scope of this article, the complexity of the phenomena has important diagnostic implications. Characterizing the turbulence requires much more than a few numbers. For the low-density case of Fig. 29, the frequencies are clearly increasing with $k$. One can infer an average dispersion relation $\bar{\omega}(k)$, but the large frequency width of the spectra at each $k$ is not consistent with an origin from linear modes with a unique $\omega(k)$. The phase velocity of the fluctuations, $v_{\mathrm{ph}}=\bar{\omega} / k$ is largely caused by the Doppler shift of plasma rotation, and it is consistent with that inferred from the plasma radial electric field, as from Fig. 18. In the plasma rest frame, $\Delta \omega \sim \bar{\omega}$, very strong frequency broadening. The modes in this case are seen to be generally of the drift wave type, but not with the idealized dispersion relation of Eq. (2.5). The results at higher density are even more complicated, with two distinct modes present.

In principle, collective scattering would be an ideal diagnostic, except for one accident of implementation. As with interferometry, the frequency $\omega_{i}$ must be chosen well above the plasma frequency for satisfactory propagation, but not too high or the scattering effect vanishes. For laboratory plasmas, this places $\omega_{i}$ in the far infrared, and the scattering angles of interest are not large. As a consequence, especially at small $k$, the viewing volume, the intersection of input and scattered beams, is not well localized within the plasma. The technique often has limited spatial resolution in practice, and the variation in detected volume with $\Delta \mathbf{k}$ makes it difficult to extract an accurate full spectrum $S(k, \omega)$. Nevertheless, it provides the best characterization of the mode(s) present in the plasma.

Complementary methods measure $\tilde{n}\left(\omega_{w}, r\right)$, the spatial localization being provided by crossed-beam techniques. One approach uses the heavy-ion-beam probe (cf. Sec. IV.D). The

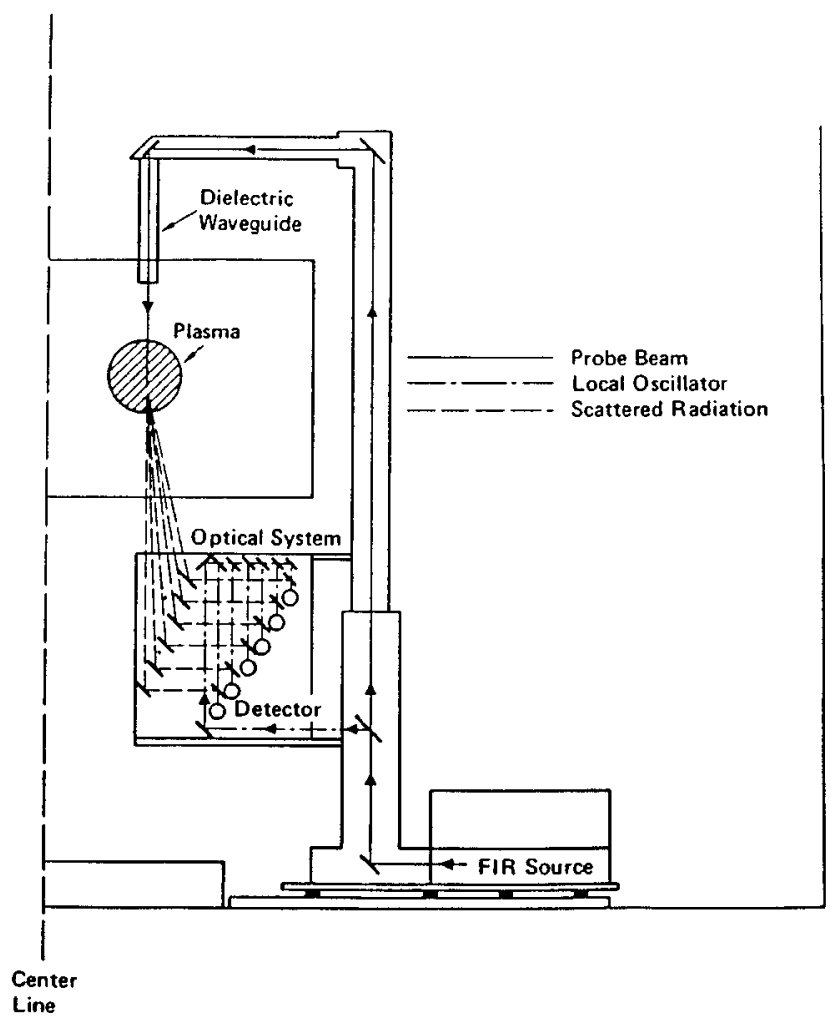

FIG. 28. Schematic of the FIR (far infrared) scattering system on TEXT. The different scattering angles correspond to different $k$ values of the density fluctuations. The entire FIR system can be moved horizontally and vertically to change the plasma volume from which the scattering originates (Brower, 1995).

secondary ion current is proportional directly to the local plasma density so that $\tilde{I} / I=\tilde{n} / n$ with little calibration needed. The spatial localization can be quite good, with sample volume dimensions of order a centimeter; but in averaging over the sample volume, it cuts out large $k_{w}$. An example of the spatial variation of the rms densityfluctuation amplitude from this diagnostic on TEXT is shown in Fig. 30 (Forster et al., 1994). Frequency spectra can easily be extracted, but $k$ information can only be obtained from the correlation of nearby sample volumes using statistical techniques. Measurable $k$ 's are even smaller than set by the sample volume limit, and complex $S(k, \omega)$, like those of Fig. 29 at high density, are not resolvable. A different technique, but with very similar characteristics, is BES (beam emission spectroscopy), which is closely related to the diagnostic neutral-beam techniques mentioned in Sec. IV.C. For this purpose, radiation from excited beam atoms is observed. The intensity is proportional to local density, the origin of the excitation, and $\tilde{n} / n$ is obtained fairly directly. The Doppler shift isolates radiation from the beam neutrals, and the crossed beam and observation paths provide localization. An example of results from TFTR is shown in Fig. 31 (Durst et al., 1993).

Reflectometry also provides information on density fluctuations. Density variations impose a phase/frequency modulation on the reflected signal and scatter radiation at small angles with respect to direct reflection. The effects are dominated by the fluctuations near the cutoff layer, $\omega=\omega_{p e}(n)$, 
K. W. Gentle: Diagnostics for magnetically confined high-temperature plasmas

(a) $\bar{n}_{\theta}=2 \times 10^{19} \mathrm{~m}^{-3}$

(b) $\bar{n}_{e}=7.5 \times 10^{19} \mathrm{~m}^{-3}$
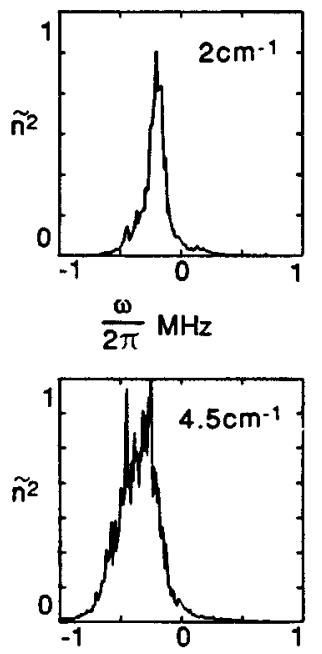

$\frac{\omega}{2 \pi} \mathrm{MHz}$
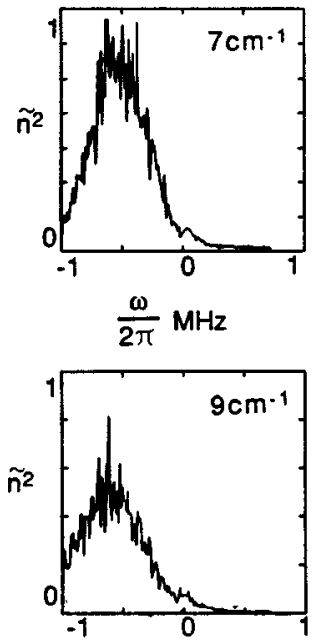

$\frac{\omega}{2 \pi} \mathrm{MHz}$

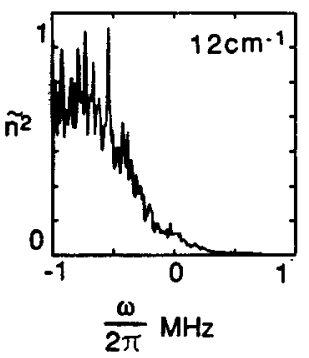

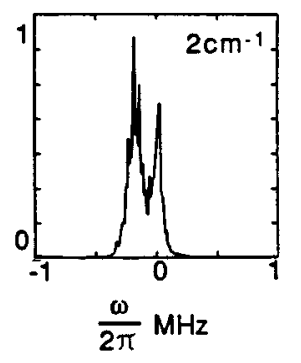

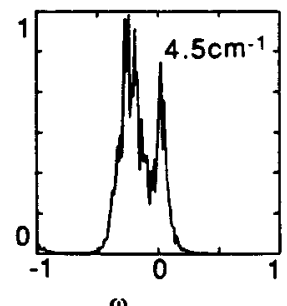

$\frac{\omega}{2 \pi} \mathrm{MHz}$

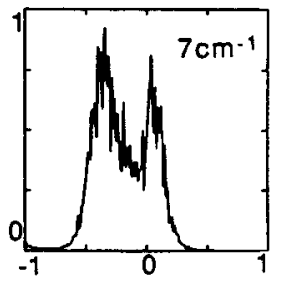

$\frac{\omega}{2 \pi} \mathrm{MHz}$

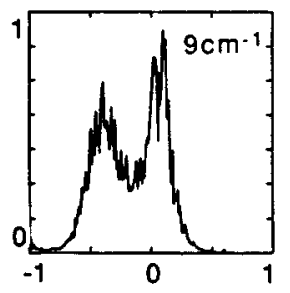

$\frac{\omega}{2 \pi} \mathrm{MHz}$

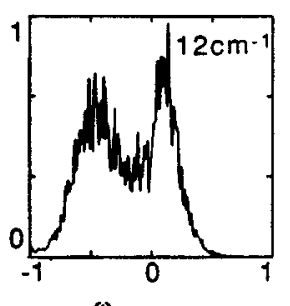

$\frac{\omega}{2 \pi} \mathrm{MHz}$

FIG. 29. Typical frequency spectra at various $k_{\theta}$ values from TEXT at two densities. The heterodyne detection system distinguishes direction of propagation through the sign of frequency (Brower, 1995).

giving good spatial localization. The technique is especially valuable for showing the positions and times of changes in turbulence (Doyle et al., 1991). There are several forms of reflectometry for fluctuation measurements, but the physics and quantitative interpretation are subtle and beyond the scope of this article [Costley, 1991(b); Mazzucato and Nazikian, 1993; Nazikian and Mazzucato, 1995].

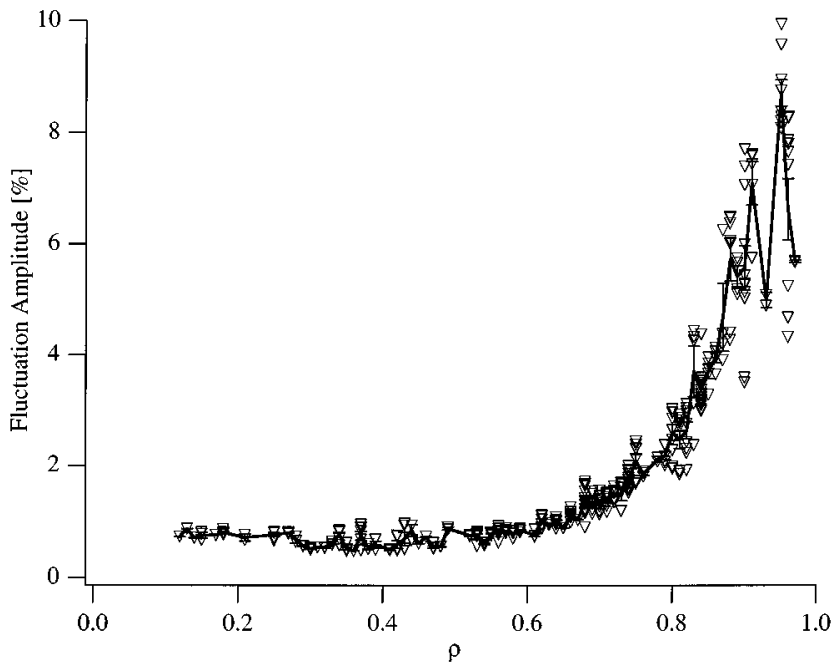

FIG. 30. Radial profile of the rms level of density fluctuations in TEXT. The level always rises toward the edge, just as the transport coefficients (cf. Fig. 22; Schock, 1995).

\section{B. Other}

Although temperature fluctuations are critical to estimating the energy transport from turbulence [cf. Eq. (2.7)], measurements of $\tilde{T}_{e}$ in the plasma interior have only recently become available. Although ECE systems have been capable of high-quality $T_{e}(r, t)$ data for some years, there is a subtle physical problem in using the technique for fluctuation mea-

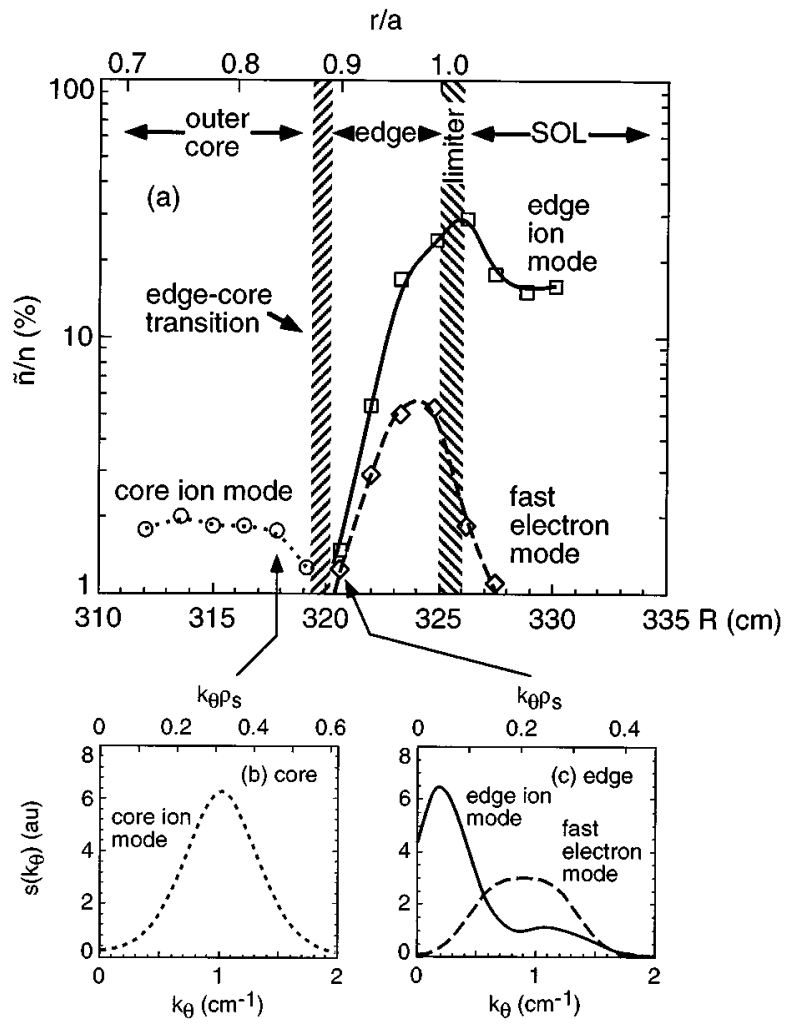

FIG. 31. Density fluctuations in the outer regions of TFTR from beam emission spectroscopy (Durst et al., 1993). (SOL is the scrape-off layer.) 


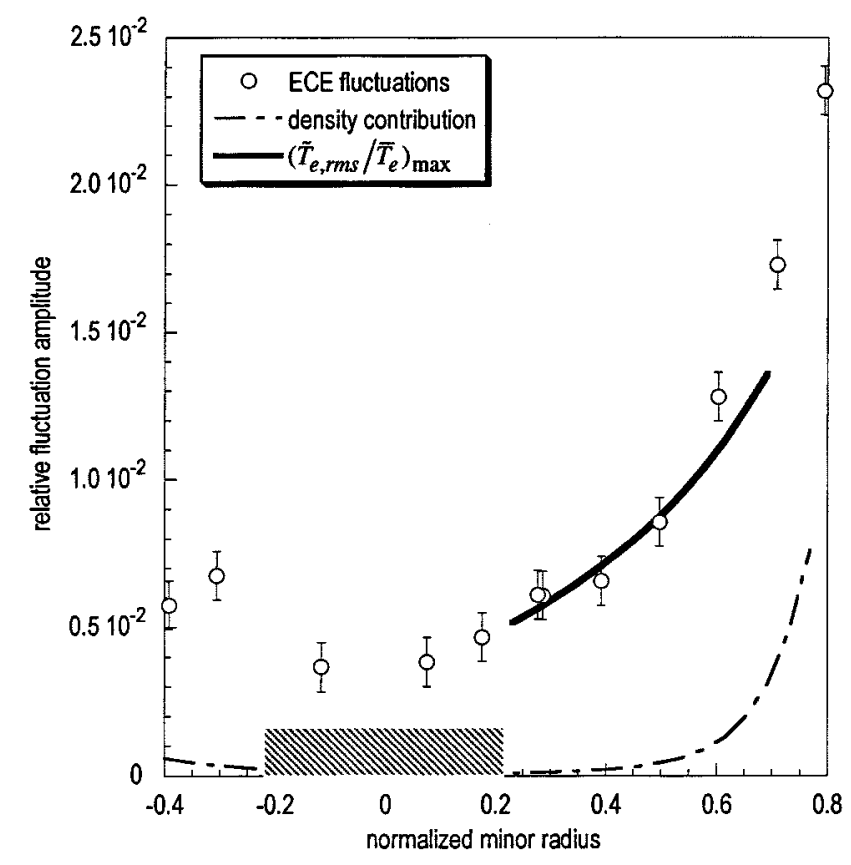

FIG. 32. Radial profile of the rms level of electron temperature fluctuations in TEXT from ECE correlations. Density fluctuations can contribute to the signal in the edge region where opacity is lower. The dashed curve is an upper bound for the effect; the heavy, solid curve shows the minimum level of temperature fluctuations (Cima, 1995).

surements. Since the ECE is blackbody radiation, it includes intrinsic intensity fluctuations at all frequencies. This effect is circumvented by ingenious correlation procedures on the ECE signals (Cima, 1992; Sattler and Hartfuss, 1993). The ECE in two statistically independent ranges (separated in frequency or emission direction) from the same physical location are correlated to extract even low levels of $\tilde{T}_{e}$, which must be common in the two ranges, from the high, but independent, intrinsic fluctuation levels of blackbody radiation in both channels. The fluctuation data obtained share many characteristics with those from the HIBP and BES techniques in giving a $\tilde{T}_{e}(r, \omega)$ with good spatial localization but a limitation to low $k$. Some recent measurements of temperature fluctuations in TEXT are shown in Fig. 32 (Cima et al., 1995).

Potential fluctuations are important as a means of inferring the $\tilde{E}_{\theta}$ that appears in Eqs. (2.6) and (2.7). The only technique is the HIBP, which can measure both $\phi$ and $\tilde{\phi}$. However, both $\tilde{\phi}$ and a $k_{\theta}$ must be measured to infer $\tilde{E}_{\theta}$.

The remaining important quantity in Eqs. (2.6) and (2.7) is $\tilde{b}_{r}$. There are no techniques at present for measuring $\tilde{b}_{r}$ in the plasma interior, nor the localized $\tilde{j}$ which produces it. The only direct measurement, which is more in the nature of a bound, is that of $\tilde{b}_{r}$ at the plasma edge. Since the $\tilde{j}$ must originate on rational magnetic surfaces, $\tilde{b}_{r}$ satisfies $\nabla \cdot \mathbf{B}=0$ and $\nabla \times \mathbf{B}=0$ from there to the edge, giving a simple relation between $\tilde{b}_{r}$ in the interior and edge. The result is that short wavelengths-higher $k$ or $m$ modes-in the interior would not be detectable outside the plasma, whereas low $m$ modes $(m \sim 6)$ of significant amplitude in the interior would be seen outside. In the absence of diagnostics for $\tilde{b}_{r}$ in the interior, indirect techniques have been employed. For example, energetic electrons-suprathermals or runaways-are not influenced by the first term in Eq. (2.6). The suprathermal density is uncoupled from $\tilde{n}$, and $\tilde{E}_{\theta}$ does not impose a random walk on the fast electrons. Their transport is largely a measure of the $\tilde{b}_{r}$ term. Various techniques for inferring the radial transport of suprathermal electrons are thus used to infer $\tilde{b}_{r}$.

\section{Transport}

Although many of the fluctuating quantities in Eqs. (2.6) and (2.7) can be measured, the appropriate combinations in the plasma interior cannot. Present diagnostics suffice for two purposes. The first is to characterize the amplitude and nature of the fluctuations present. This serves as a guide, constraint, and check on theories. If we had complete theories for the turbulent transport of particles and energy, present diagnostics would be capable of examining the predictions of turbulence critically. Even if the theories were incomplete, present diagnostics applied to well-designed experiments would probably be sufficient to determine one or two unknown parameters and still test the theory.

The second purpose is to provide bounds and estimates of transport. Even if all the terms are not known, many of the important magnitudes are now measurable. Particularly in the context of certain classes of theories, reasonable estimates of turbulent transport can be obtained for comparison with experiment. For example, if the magnitudes of density and electric-field fluctuations are known, an upper bound for the first term in Eq. (2.6) is established; lack of correlation can only decrease the fluctuation-driven transport.

\section{KINETIC EFFECTS}

Kinetic effects are pervasive in hot plasmas. Even though the fluid description is used as the basic framework, kinetic equations are required for many important analyses, and the fluid equations are often supplemented with kinetic results. the consequences of these calculations are often tested in experiment, but direct experimental techniques for examining the distribution functions are limited. The ion distribution function can be examined using charge-exchange neutrals as discussed in Sec. IV.C. (The tail is quite accessible; the principal uncertainty is the subthermal population and possible presence of a cold population near the edge.) Less information is available for electrons. Very energetic $(\mathrm{MeV})$ electrons ("runaways") can be detected from the gamma rays produced, and the presence of suprathermals is evidenced in ECE at levels above blackbody, especially at harmonics of the cyclotron frequency, and conversely in absorption (Kirkwood et al., 1990; Skiff et al., 1993). Measurement of X-ray bremsstrahlung spectrum in the range $5-15 \mathrm{keV}$ with standard techniques also provides some information on the tail of the electron distribution function. None of the techniques measures $f(v)$. Rather, they suffice to determine a few parameters in a model $f(v)$ with some sort of non-Maxwellian tail. 


\section{ASYMMETRIES AND EDGE EFFECTS}

The diagnostic implications of asymmetries are largely indirect. The use of a diagnostic is rarely prevented by asymmetries, but the usefulness and interpretation may be greatly changed. In the worst cases, the chord-integrated diagnostics like density, symmetry is essential to inferring local parameters. A lack of symmetry would require many additional chords to permit inversion, a requirement which may not be feasible in practice. Even the prototypical local measurement, Thomson scattering for $T_{e}$, relies on symmetry to minimize the number of points at which measurement must be made. Much more data are required to characterize an asymmetric plasma.

This has an important impact on the measurement of quantities that are not flux-surface quantities and might therefore be expected to have intrinsic asymmetries. All fluctuating quantities are of this sort. Exploration of asymmetries is not precluded in principle, but the access required by most techniques prevents their use over the full cross section in practice.

Specialized diagnostics for the plasma edge are generally beyond the scope of this review. On one hand, the lower temperatures and proximity to the wall expand the range of techniques. On the other hand, the interactions with the wall lack symmetry - toroidal, poloidal, or both. Therefore much more extensive measurements are necessary to characterize the region. One aspect of edge diagnostics should be mentioned, however, because of its significance for corroborating interior measurements. Conventional Langmuir probe techniques (e.g., Hutchinson, 1987) can be applied in the edge, even measuring all quantities for the electrostatic contribution to transport in Eq. (2.6). Probe measurements have been used to confirm levels of $\tilde{n}$ from scattering measurements (cf. Sec. VI.A) by Ritz et al. (1987) and to demonstrate that the electrostatic particle flux from Eq. (2.6) largely accounts for particle transport at the edge (Rowan et al., 1987), thus emphasizing the importance of turbulence measurements.

\section{CONCLUSION}

Measuring techniques for hot plasmas have improved dramatically over the last decade. Instead of characterizing plasmas with no more than a few parameters in a zerodimensional representation, tokamak plasmas are now routinely described by flux surfaces and the full onedimensional variation of fluid quantities on flux surfaces. The experimental techniques are sufficient to confirm most aspects of the basic fluid representation for equilibrium and stability and to explore the rich physics of plasma transport in considerable detail.

\section{ACKNOWLEDGMENTS}

The author would like to thank D. V. Bartlett, D. L. Brower, G. Cima, A. E. Costley, R. D. Durst, P. C. Efthimion, B. Grek, R. Groebner, I. H. Hutchinson, L. L. Lao, F. M. Levinton, S. S. Medley, C. Petty, P. M. Schoch, S. D. Scott, P. E. Stott, E. J. Synakowski, C. Watts, K. M. Young, and M. C. Zarnstorff for providing references and figures, in many cases especially adapted for this review. This work was supported by the U.S. Department of Energy under Grant No. DE-FG05-88ER-53267.

\section{REFERENCES}

Bartlett, D. V., 1995, private communication.

Bartlett, D. V., et al., 1988, Nucl. Fusion 28, 73.

Bartlett, D. V., A. E. Costley, L. Porte, R. Prentice, N. A. Salmon, and G. Sips, 1990, J. Nucl. Mater. 176, 1064.

Bateman, G., 1978, MHD Instabilities (MIT, Cambridge, MA).

Bekefi, G., 1966, Radiation Processes in Plasmas (Wiley, New York).

Bornatici, M., R. Cano, O. De Barabieri, and F. Engelmann, 1983, Nucl. Fusion 23, 1153.

Brower, D. L., 1995, private communication.

Brower, D. L., Y. Jiang, W. A. Peebles, S. Burns, and N. C. Luhmann, Jr., 1992, Rev. Sci. Instrum. 63, 4990.

Brower, D. L., S. K. Kim, W. A. Peebles, and N. C. Luhmann, Jr., 1988, Rev. Sci. Instrum. 59, 1559.

Brower, D. L., W. A. Peebles, and N. C. Luhmann, Jr., 1987, Nucl. Fusion 27, 2055.

Burrell, K. H., K. W. Gentle, N. C. Luhmann, Jr., E. S. Marmar, M. Murakami, K. F. Schoenberg, W. M. Tang, and M. C. Zarnstorff, 1990, Phys. Fluids B 2, 2904.

Cima, G., 1995, private communication.

Cima, G., 1992, Rev. Sci. Instrum. 69, 4630.

Cima, G., T. D. Rempel, R. V. Bravenec, R. F. Gandy, M. Kwon, C. Watts, and A. J. Wootton, 1995, Phys. Plasmas (in press).

Cormack, A. M., 1964, J. Appl. Phys. 10, 2908.

Costley, A. E., 1988, Plasma Phys. Contr. Fusion 30, 1455.

Costley, A. E., 1991a, in Diagnostics for Contemporary Fusion Experiments, edited by P. E. Stott, D. K. Akulina, G. Gorini, and E. Sindoni (Editrice Compositori, Bologna), p. 223.

Costley, A. E., 1991b, in Diagnostics for Contemporary Fusion Experiments, edited by P. E. Stott, D. K. Akulina, G. Gorini, and E. Sindoni (Editrice Compositori, Bologna), p. 113.

Crowley, T. P., 1994, Plasma Sci. 22, 291.

Doyle, E. J., R. J. Groebner, K. H. Burrell, P. Gohil, T. Lehecka, N. C. Luhmann, Jr., H. Matsumoto, T. H. Osborne, W. A. Peebles, and R. Philipona, 1991, Phys. Fluids B 3, 2300.

Durst, R. D., R. J. Fonck, J. S. Kim, S. F. Paul, N. Bretz, C. Bush, Z. Chang, and R. Hulse, 1993, Phys. Rev. Lett. 71, 3135.

Efthimion, P. C., 1995, private communication.

Efthimion, P. C., et al., 1991, Phys. Rev. Lett. 66, 421.

Fonck, R. J., D. S. Darrow, and K. P. Jaehnig, 1984, Phys. Rev. A 29, 3288.

Forster, J. D., P. M. Schoch, R. L. Hickok, and W. C. Jennings, 1994, Plasma Sci. 22, 359.

Freidberg, J. P., 1987, Ideal Magnetohydrodynamics (Plenum, New York).

Gentle, K. W., 1988, Phys. Fluids 31, 1105.

Gentle, K. W., O. Gehre, and K. Krieger, 1992, Nucl. Fusion 32, 217.

Gentle, K. W., et al., 1995, Phys. Rev. Lett. 74, 3620.

Gohil, P., K. H. Burrell, R. J. Groebner, and R. P. Seraydarian, 1990, Rev. Sci. Instrum. 61, 2949.

Goldston, R. J., D. C. McCune, H. H. Towner, S. L. Davis, R. J. Hawryluk, and G. L. Schmidt, 1981, J. Comput. Phys. 43, 61.

Gorini, G., P. Mantica, G. M. D. Hogeweij, F. DeLuca, A. Jacchia, J. A. Lonigs, N. J. Lopes Cardozo, and M. Peters, 1993, Phys. Rev. Lett. 71, 2038.

Grad, H., 1967, Phys. Fluids 10, 137. 
Granetz, R. S., and P. Smeulders, 1988, Nucl. Fusion 28, 457.

Grek, B., 1995, private communication.

Groebner, R. J., 1994, private communication.

Groebner, R. J., N. H. Brooks, K. H. Burrell, and L. Rottler, 1983, Appl. Phys. Lett. 43, 920.

Hazeltine, R. D., and J. D. Meiss, 1992, Plasma Confinement (Addison-Wesley, Redwood City, CA).

Hinton, F. L., and R. D. Hazeltine, 1976, Rev. Mod. Phys. 48, 239.

Howard, J., E. J. Doyle, G. Reibeiz, R. L. Savage, Jr., W. A. Peebles, and N. C. Luhmann, Jr., 1988, Rev. Sci. Instrum. 59, 2135.

Hutchinson, I. H., 1987, Principles of Plasma Diagnostics (Cambridge University, Cambridge, England).

Hutchinson, I. H., et al., 1994, Phys. Plasmas 1, 1511.

Isler, R. C., L. E. Murray, S. Kasoli, J. L. Dunlap, S. C. Bate, P. H. Edmonds, E. A. Lazarus, C. M. Ma, and M. Murakami, 1981, Phys. Rev. A 24, 2701.

Jahns, G. L., M. Soler, B. V. Wadell, J. D. Callen, and H. R. Hicks, 1978, Nucl. Fusion 18, 609.

JET Team, 1992, Nucl. Fusion 32, 187.

Johnson, D., D. Dimock, B. Grek, D. Long, D. McNeil, R. Palladino, J. Robinson, and E. Tolnas, 1985, Rev. Sci. Instrum. 56, 1015.

Kadomtsev, B. B., and O. P. Pogutse, 1975, in Reviews of Plasma Physics, edited by M. A. Leontovich (Consultants Bureau, New York), Vol. 5, p. 249.

Kirkwood, R. K., I. H. Hutchinson, S. C. Luckhardt, and J. P. Squire, 1990, Nucl. Fusion 30, 431.

Kissick, M. W., E. D. Fredrikson, J. D. Callen, C. E. Bush, Z. Chang, P. C. Efthimion, R. A. Hulse, D. K. Mansfield, H. K. Park, J. F. Schivell, S. D. Scott, E. J. Synakowski, G. Taylor, M. C. Zarnstorff, 1994, Nucl. Fusion 34, 349.

Krall, N. A., and A. W. Trivelpiece, 1973, Principles of Plasma Physics (McGraw-Hill, New York).

Kruskal, M. D., and R. M. Kulsrud, 1958, Phys. Fluids 1, 265.

Lao, L. L., 1995, private communication.

Lao, L. L., H. St. John, R. D. Stambaugh, A. G. Kellman, and W. Pfeiffer, 1985, Nucl. Fusion 25, 1611.

Levinton, F. M., 1992, Rev. Sci. Instrum. 63, 5157.

Levinton, F. M., S. H. Batha, M. Yamada, and M. C. Zarnstorff, 1993, Phys. Fluids B 5, 2554.

Liewer, P. C., 1985, Nucl. Fusion 25, 543.

Manheimer, W. M., and C. N. Lashmore-Davies, 1989, MHD and Microinstabilities in Confined Plasma (Hilger, Bristol).

Mansfield, D. K., H. K. Park, L. C. Johnson, H. M. Anderson, R. Chauimard, V. S. Foote, C. M. Ma, and B. J. Clifton, 1987, Appl. Opt. 26, 4469.

Marmar, E. S., J. L. Terry, B. Lipshultz, and J. E. Rice, 1989, Rev. Sci. Instrum. 60, 3739.

Mast, K. F., and H. Krause, 1985, Rev. Sci. Instrum. 56, 969.
Mazzucato, E., and R. Nazikian, 1993, Phys. Rev. Lett. 71, 1840.

Muller, E. R., K. Behringer, and H. Niedermeyer, 1982, Nucl. Fusion 22, 1651.

Nazikian, R., and E. Mazzucato, 1995, Rev. Sci. Instrum. 66, 392. Park, H. K., 1989, Plasma Phys. Contr. Fusion 31, 2035.

Petty, C. C., 1995, private communication.

Pfeiffer, W. W., F. B. Marcus, C. J. Armentraut, G. L. Jahns, J. W. Petrie, and R. E. Stockdale, 1985, Nucl. Fusion 25, 655.

Rice, B. W., and E. B. Hooper, 1994, Nucl. Fusion 34, 1.

Ritz, Ch. P., et al., 1987, Nucl. Fusion 27, 1125.

Roquemore, A. L., G. Gammel, G. W. Hammett, R. Kaita, and S. S. Medley, 1985, Rev. Sci. Instrum. 56, 1120.

Rowan, W. L., et al., 1987, Nucl. Fusion 27, 1105.

Salzmann, H., J. Bundgaard, A. Gadd, C. Gowers, K. B. Hansen, K. Hirsch, P. Nielsen, K. Reed, C. Schrödter, and K. Weisberg, 1988, Rev. Sci. Instrum. 59, 1451.

Sattler, S., and H. J. Hartfuss, 1993, Plasma Phys. Contr. Fusion 35, 1285.

Schivell, J., 1985, Rev. Sci. Instrum. 56, 972.

Schock, P. M., 1995, private communication.

Scott, S. D., et al., 1990, Phys. Rev. Lett. 64, 531.

Shafranov, V. D., 1966, in Reviews of Plasma Physics, edited by M. A. Leontovich (Consultants Bureau, New York), Vol. 2, p. 103.

Sheffield, J., 1975, Plasma Scattering of Electromagnetic Radiation (Academic, New York).

Skiff, F., D. A. Boyd, and J. A. Colborn, 1993, Phys. Fluids B 5, 2445.

Soltwisch, H., 1988, Rev. Sci. Instrum. 59, 1599.

Stott, P. E., 1992, Rep. Prog. Phys. 55, 1715.

Stott, P. E., D. K. Akulina, G. Gorini, and E. Sindoni, 1991, Eds., Diagnostics for Contemporary Fusion Experiments (Editrice Compositori, Bologna).

Strachan, J. D., et al., 1994, Phys. Rev. Lett. 72, 3526.

Synakowski, E. J., et al., 1993, Phys. Fluids B 5, 2215.

Tang, W. M., 1978, Nucl. Fusion 18, 1089.

Towner, H. H., R. J. Goldston, G. W. Hammett, J. A. Murphy, C. K. Phillips, S. D. Scott, M. C. Zarnstorff, and D. Smithe, 1992, Rev. Sci. Instrum. 63, 4753.

Ware, A. A., 1970, Phys. Rev. Lett. 25, 15.

Wen, Y., and R. V. Bravenec, 1995, Rev. Sci. Instrum. 66, 549.

Wesson, J., 1987, Tokamaks (Oxford University Press, Oxford).

West, W. P., D. M. Thomas, J. S. DeGrassie, and S. B. Zheng, 1987, Phys. Rev. Lett. 58, 2758.

Wootton, A. J., B. A. Carreras, H. Matsumoto, K. McGuire, W. A. Peebles, Ch. P. Ritz, P. W. Terry, and S. J. Zweben, 1990, Phys. Fluids B 2, 2879.

Zarnstorff, M. C., 1995, private communication.

Zarnstorff, M. C., K. McGuire, M. G. Bell, B. Grek, D. Johnson, D. McCune, H. Park, A. Ramsey, and G. Taylor, 1990, Phys. Fluids B 2, 1852. 